\title{
Revisiting Laws of Black Hole Mechanics and Violation of Null Energy Condition
}

\author{
Susobhan Mandal \\ Department of Physical Sciences, Indian Institute of Science Education and Research Kolkata, Mohanpur, India \\ Email: sm17rs045@iiserkol.ac.in
}

How to cite this paper: Mandal, S. (2019) Revisiting Laws of Black Hole Mechanics and Violation of Null Energy Condition. Journal of High Energy Physics, Gravitation and Cosmology, 5, 82-111. https://doi.org/10.4236/jhepgc.2019.51004

Received: October 10, 2018

Accepted: December 3, 2018

Published: December 6, 2018

Copyright (C) 2019 by author and Scientific Research Publishing Inc. This work is licensed under the Creative Commons Attribution International License (CC BY 4.0).

http://creativecommons.org/licenses/by/4.0/

\begin{abstract}
Most of the important and powerful theorems in General Relativity such as singularity theorems and the theorems applied for null horizons depend strongly on the energy conditions. However, the energy conditions on which these theorems are based on, are beginning to look at less secure if one takes into accounts quantum effects which can violate these energy conditions. Even there are classical systems that can violate these energy conditions which would be problematic in validation of those theorems. In this article, we revisit to a class of such important theorems, the laws of black hole mechanics which are meant to be developed on null like killing horizons using null energy condition. Then we show some classical and quantum mechanical systems which violate null energy condition based on which the above theorem stands.
\end{abstract}

\section{Keywords}

Black Hole, Null Energy Condition, Quantum Mechanical Systems

\section{Introduction}

General Relativity, one of the successful theory in modern physics which describes gravity successfully in terms of introducing the concepts of spacetime manifolds, is often considered to be tremendously complex theory when one is looking for solutions of Einstein equation

$$
G_{\mu v}=\frac{8 \pi G}{c^{4}} T_{\mu v}
$$

Left hand side of this equation comes from description of geometry of spacetime manifolds which by itself is complicated covariant tensor of rank 2 but it is at least universal function of spacetime geometry. On the other hand, right 
hand side describes the matter part of theory which is not universal at all rather it depends upon our choice. Based on above one can have two choices of which first is to do special-case calculations, one for each conceivable matter action or second is develop general theorems based on some generic features which reasonably all stress-energy should satisfy.

One such feature that most matter seems to share (found mostly through experiments) is that energy densities (almost) always seem to be positive. Energy conditions in General Relativity are a variety of different ways of imposing the fact that energy density is positive definite locally. The energy conditions basically say that what are the possible linear combination of components of stress-energy tensor at any specified point would be positive.

Almost all the powerful theorems in General Relativity requires some form of energy condition (some notion of positivity of stress-energy tensor) as an input hypothesis and the variety of such energy conditions have been used in community driven largely based on how easily one can prove certain theorems.

Due to the progress of Quantum Field Theory in curved spacetime, people started realizing that quantum matter in classical geometry often violates various energy conditions. But still since violation of these energy conditions arise due to quantum effects which are typically proportional to $\hbar$, sometimes people don't take it seriously [1]. It has also become clear that there are in fact classical field theories [2] [3] that violate energy condition but compatible with all known experiments. Because these are now classical violations of energy conditions they can be made arbitrarily large.

In this article, our goal is to show how laws of black hole thermodynamics or more specifically four theorems on null killing horizons strongly depends on null energy condition. To do that we systematically develop the mathematical idea to reach at those theorems and give their proofs.

Then we look at some examples of both classical and quantum field theories where null energy condition is actually violated. And we also comment on why these features of stress-energy tensor is not universal. This directly concludes that above theorems are weakly valid and any spacetime whose source stressenergy tensor violates null energy condition for them such theorems does not hold unless statements of the theorems are suitably corrected.

\section{Energy Conditions}

Before proceeding further towards the mathematical development for the said theorems, we first look at all possible energy conditions, their statements and their current status in physics.

To familiar with basic nomenclature, the pointwise energy conitions often used in General relativity are [4] [5]:

- Trace energy condition (TEC) which states that trace of stress-energy tensor at most equal to zero which mathematically states $T=g_{\mu v} T^{\mu \nu} \leq 0$

- Strong energy condition (SEC) which states that for every future-pointing 
timelike vector field $\boldsymbol{X}$, the trace of the tidal tensor measured by the corresponding observers is always non-negative which in mathematics is to say $\left(T_{\mu \nu}-\frac{1}{2} g_{\mu \nu} T\right) X^{\mu} X^{v} \geq 0$.

- Null energy condition (NEC) which states that for every future-pointing null vector field $\boldsymbol{n}$, the quantity $T_{\mu \nu} n^{\mu} n^{v} \geq 0$.

- Weak energy condition (WEC) which states that for every future-pointing timelike vector field $\boldsymbol{X}$ the matter density observed by the corresponding observers is always non-negative which in mathematical terms simply means that $T_{\mu v} X^{\mu} X^{v} \geq 0$

- Dominant energy condition (DEC) which states that in addition to the weak energy condition holding true, for every future-pointing causal vector field (either timelike or null) $\boldsymbol{Y}$, the vector field $-T_{v}^{\mu} Y^{v}$ must be a futurepointing causal vector. In other words to say, mass-energy can never be observed to be flowing faster than light.

The above conditions are often described in terms of considering a perfect fluid as the chosen matter of the system whose stress-energy tensor is $T^{\mu \nu}=(\rho+P) u^{\mu} u^{\nu}+P g^{\mu \nu}$ (in $c=\hbar=1$ unit) where fluid is at rest in comoving coordinates. Then one can write above conditions as follows

- Trace energy condition (TEC): $-\rho-3 P \geq 0$.

- Strong energy condition (SEC): $-\rho+3 P \geq 0$.

- Null energy condition (NEC): $-\rho+P \geq 0$.

- Weal energy condition (WEC): $-\rho \geq 0, p \geq 0, \rho+P \geq 0$.

- Dominant energy condition (DEC): $-\rho \geq 0, P \in[-\rho, \rho]$

Then, these are linear relationships or rather inequalities between the energy density and the pressure of the matter of fields that is believed to generate the spacetime curvature.

Violations of these energy conditions have often been treated as only being produced by unphysical stress energy tensors. If the null energy condition is violated, and then weak energy condition is violated as well in some system, then negative energy densities and so negative masses are thus physically admitted. However, although the energy conditions are widely used to prove theorems concerning singularities and black holes thermodynamics, such as the area increase theorem, the topological censorship theorem, and the singularity theorem of stellar collapse as presented by Visser (1996) they really lack a rigorous proof from fundamental principles. Moreover, several situations in which they are violated are known, perhaps the most quoted being the Casimir effect [6] [7]. Although observed violations are produced by small quantum systems, resulting in the order of $\hbar$ but it can be used to eliminate certain energy conditions.

One particular energy condition, the trace energy condition has been completely abandoned and forgotten. The trace energy condition says that the trace of stress-energy tensor must always be negative or positive depending on metric conventions, and was popular for a while during the 1960s. However, once it was found that stiff equations of state, such as those for neutron stars, 
violate the trace energy condition, this energy condition fell into disfavour. It has been now completely abandoned and is no longer put as example in literature.

This is also general agreement that the strong energy condition is dead: 1) The most naive scalar field theory we may write down, the minimally coupled scalar field, violates the strong energy condition and indeed curvature-coupled scalar field theories also violate the strong energy condition. The specific models of point-like particles with two-body interactions also violate the strong energy condition [8]. 2) The strong energy condition must be violated during the inflationary epoch [9], and need for this strong energy condition violation is why inflationary models are typically driven by scalar inflation fields. 3) The observational data regarding the accelerating Universe, the strong energy conditions are violated on cosmological scales.

Over the last decade, or so it has started becoming obvious that quantum effects are capable of violating all the energy conditions, even the weakest of the standard energy conditions. Despite the fact that they are moribund, because of the lack of successful replacements, the null energy conditions, weak energy conditions, and dominant energy conditions are still extensively used in general relativity. The weakest of these is the null energy condition, and it is in many cases also the easiest one to work with and analyse.

The aim of this article is to show by through laws of black hole thermodynamics that how strong statements of such powerful theorems depends completely on the existence of such energy conditions. And we want to break the standard wisdom for many years which is that all reasonable forms of matter should at least satisfy the null energy condition through showing some examples where quantum effects can indeed show violation of such energy conditions and even in classical cases also we have found such violations.

\section{Geometry of Null Hypersurfaces}

\subsection{Introduction to Null Hypersurfaces}

Since black hole event horizon is a null hyperrsurface, we should go through the geometry of null-hypersurfaces. The mathematical definitions and ideas are mainly based on [10] [11] [12].

So let us first recall what are hypersurfaces of a manifold $(\mathcal{M}, g)$ where $\underline{g}$ is the metric of the spacetime $\mathcal{M}$. So a hypersurface is an embedded manifold of $\mathcal{M}$ of codimension 1 .

And this leads us to our next definition which is, what are null hypersurfaces. On a Lorentzian manifold $(\mathcal{M}, \underline{g})$, a hypersurface $\Sigma$ can locally be classified into 3 categories of which null-hypersurface is one and this classification depends on the type of metric induced by $\underline{g}$ on $\Sigma$ which is nothing but the restriction $\left.\underline{g}\right|_{\Sigma}$ to $\underline{g}$ of vector fields tangent to $\Sigma$. And a hypersurface $\Sigma$ is said to be null-hypersurface iff $\left.\underline{g}\right|_{\Sigma}$ is degenerate and that is iff $\left.\operatorname{sign} \underline{g}\right|_{\Sigma}=(0,+,+)$. Null hypersurfaces have a distinctive feature which is that their normals are also tangent to them according to the definition of null vector. 
hypersurface as level sets:-As any hypersurface $\mathcal{H}$ can be locally considered as a level set: around any point of $\mathcal{H}, \exists$ an open subset $\mathcal{U}$ of $\mathcal{M}$ and a smooth scalar field $u: \mathcal{U} \rightarrow \mathbb{R}$ such that

$$
\forall p \in \mathcal{U}, p \in \mathcal{H} \leftrightarrow u(p)=0
$$

and

$$
\underline{\nabla u} \neq 0
$$

Second condition actually ensures that $\mathcal{H}$ is a regular hypersurface and without it $\mathcal{H}$ may be self intersecting.

A very simple example of null hypersurface is a null hyperplane in 4-dimension Minkowski spacetime which are labelled by function $u(t, x, y, z)=t-x$.

From now onwards we denote null hypersurface by $\mathcal{H}$.

Null normals:-Let $\boldsymbol{l}$ be a vector field normal to $\mathcal{H}$, since $\mathcal{H}$ is a null hypersurface, $\boldsymbol{l}$ is a null vector

$$
\boldsymbol{l} \cdot \boldsymbol{l}=g_{\mu \nu} l^{\mu} l^{v}=0
$$

And we also choose $\boldsymbol{l}$ to be future-directed for mathematical convenience in later. Note that as a consequence of the definition there is no natural normalization of null vectors unlike in the case of time-like and space-like hypersurfaces. Therefore, we can always define null normal upto a scaling function which is strictly positive.

We consider null normal vector field not confined in $\mathcal{H}$ but rather defined in some open subset of $\mathcal{M}$ around $\mathcal{H}$, so that we can define spacetime covariant derivative $\nabla \boldsymbol{l}$. A simple way to achieve this is to consider not only a single hypersurface $\mathcal{H}$ but a foliation of $\mathcal{M}$ by a family of null hypersurfaces labelled by scalar field $u$, denote them as $\left(\mathcal{H}_{u}\right)$ and null hypersurface $\mathcal{H}$ is nothing but the element $\mathcal{H}_{u=0}=\mathcal{H}$.

Since $\mathcal{H}$ is a hypersurface where $u$ is constant, then by definition $\forall \boldsymbol{v} \in T_{p} \mathcal{M}, \boldsymbol{v}$ is tangent to $\mathcal{H}$

$$
\nabla_{v} u=0 \Rightarrow\langle\nabla u, v\rangle=0 \Rightarrow \nabla u \cdot v=0
$$

where $\nabla u$ is the gradient vector field of the scalar field $u$, which in index notation can be written as follows

$$
\nabla^{\alpha} u=g^{\alpha \mu} \nabla_{\mu} u=g^{\alpha \mu} \frac{\partial u}{\partial x^{\mu}}
$$

Note that property (5) implies that $\nabla u$ is normal vector field of $\mathcal{H}$. By uniqueness condition of the normal direction to hypersurface, it must be collinear to $\boldsymbol{l}$. Therefore, there must exists a scalar function $\rho$ such that

$$
\boldsymbol{l}=-\mathrm{e}^{\rho} \nabla u
$$

The minus sign ensures that in the case of $u$ increasing toward future, $l$ is future-directed.

\subsection{Null Geodesic Generators}

Consider $\boldsymbol{l}=-\mathrm{e}^{\rho} \nabla u$. Then note that using covariant derivative, anti-symmetrizing 
$\nabla_{\alpha} l_{\beta}$ and the torsion-free property of $\nabla$ (i.e. $\nabla_{\alpha} \nabla_{\beta} u-\nabla_{\beta} \nabla_{\alpha} u=0$ ), we will find

$$
\nabla_{\alpha} l_{\beta}-\nabla_{\beta} l_{\alpha}=\nabla_{\alpha} \rho l_{\beta}-\nabla_{\beta} \rho l_{\alpha}
$$

which we can write in terms of exterior derivative and wedege product of 1 -forms

$$
\mathrm{d} \underline{l}=\underline{\mathrm{d} \rho} \wedge \underline{l}
$$

This is known as Frobenius identity.

Let contract Frobenius identity with $\boldsymbol{l}$, then we will get

$$
\begin{aligned}
& l^{\mu} \nabla_{\mu} l_{\alpha}-\underbrace{l^{\mu} \nabla_{\alpha} l_{\mu}}_{=0}=l^{\mu} \nabla_{\mu} \rho l_{\alpha}-\underbrace{l^{\mu} l_{\mu}}_{=0} \nabla_{\alpha} \rho \\
& \Rightarrow l^{\mu} \nabla_{\mu} l_{\alpha}=\kappa l_{\alpha}, \kappa=l^{\mu} \nabla_{\mu} \rho \\
& \Rightarrow \nabla_{l} \underline{l}=\kappa \underline{l}, \kappa=\nabla_{l} \rho
\end{aligned}
$$

This equation shows that the field lines of $\boldsymbol{l}$ are geodesics. To demonstrate this we rescale the vector field $\boldsymbol{l} \mapsto \boldsymbol{l}^{\prime}=\alpha \boldsymbol{l}$ and we demand that $\nabla_{\boldsymbol{l}^{\prime}} \underline{\boldsymbol{l}}^{\prime}=0$. If one evaluate $\nabla_{l^{\prime}} \underline{\underline{l}}^{\prime}$ he/she will find

$$
\nabla_{l^{\prime}} \boldsymbol{l}^{\prime}=\alpha\left(\nabla_{l} \alpha+\kappa \alpha\right) \boldsymbol{l}
$$

Therefore, $\nabla_{l^{\prime}} \underline{\boldsymbol{l}}^{\prime}=0$ demands that $\nabla_{l} \ln \alpha=-\kappa$. Therefore, it suffices to solve this $1^{\text {st }}$ order differential equation to ensure that $\boldsymbol{l}^{\prime}$ is a geodesic vector field.

Because of $\nabla_{l^{\prime}} \underline{\boldsymbol{l}}^{\prime}=0$, the field lines of $\boldsymbol{l}^{\prime}$ are null geodesics and $\boldsymbol{l}^{\prime}$ is the tangent vector to them associated with some affine parameter $\lambda$. On the other hand if $\kappa \neq 0, l$ is not a geodesic vector fields and therefore we can't associate it with some affine parameter. And that's why we call $\kappa$ non-affinity coefficient of null-normal $\boldsymbol{l}$.

Since $\boldsymbol{l}$ is collinear to $\boldsymbol{l}^{\prime}$, it is obviously shares the same field lines which just have been shown to be null geodesics. These field lines are called null geodesic generators.

Any null hypersurface $\mathcal{H}$ is ruled by a family of null geodesics, called the null generators of $\mathcal{H}$ and each vector field $\boldsymbol{l}$ normal to $\mathcal{H}$ is tangent to these null geodesics.

\subsection{Cross-Sections}

A key parameter is expansion of null hypersurfaces, which we will discuss once we will go through discussion about Cross-section.

From now on we assume that spacetime dimension $\mathrm{n}$ obeys $n \geq 3$. We define then a cross-section of the null hypersurfaces $\mathcal{H}$ as a submanifold $\mathcal{N}$ of $\mathcal{H}$ of codimension 2 (i.e. $\operatorname{dim} \mathcal{N}=n-2$ ), such that 1) the null normal $\boldsymbol{l}$ is nowhere tangent to $\mathcal{N}$ and 2) each null geodesic generator of $\mathcal{H}$ intersects $\mathcal{N}$ once and only once.

Indices relative to cross-section will range from 2 to $n-1$ and will be denoted by a latin letters. 
To encompass the idea that an event horizon delimitates some region of spacetime, we shall assume that the clear the cross-sections are closed manifolds, i.e. compact without boundary. Having the definiton of a cross-section $\mathcal{N}$, the topology of $\mathcal{H}$ is $\mathbb{R} \times \mathcal{N}$. For 4-dimensional Schwarzschild black hole this is $\mathcal{H} \simeq \mathbb{R} \times \mathcal{N}$.

Without giving a detailed proof we make the statement here that any crosssection $\mathcal{N}$ is spacelike, i.e. all vectors tangent to $\mathcal{N}$ are spacelike.

Let $q$ be the metric induced on $\mathcal{N}$ by $g$, then we can write

$$
\forall(\boldsymbol{u}, \boldsymbol{v}) \in T_{p} \mathcal{N} \times T_{p} \mathcal{N}, \underline{\mathrm{q}}(\boldsymbol{u}, \boldsymbol{v})=\underline{\mathrm{g}}(\boldsymbol{u}, \boldsymbol{v})
$$

And $\mathcal{N}$ is spacelike is equivalent to saying that metric $\mathrm{q}$ is positive-definite and $(\mathcal{N}, \underline{\mathrm{q}})$ is a Riemannian maniold.

An important consequence of $\mathcal{N}$ being spacelike is that at each point $\mathcal{N}$ the tangent space $T_{p} \mathcal{N}$ has an orthogonal complement $T_{p}^{\perp} \mathcal{N}$ which is a timelike hyperplane such that $T_{p} \mathcal{M}$ is a direct sum of $T_{p} \mathcal{N}$ and $T_{p}^{\perp} \mathcal{N}$ :

$$
\forall p \in \mathcal{N}, T_{p} \mathcal{M}=T_{p} \mathcal{N} \oplus T_{p}^{\perp} \mathcal{N}
$$

And the metric induced by $g$ on $T_{p}^{\perp} \mathcal{N}$ is Lorentzian.

Note that since $\mathcal{N} \subset \mathcal{H}$, therefore, the null normal $\boldsymbol{l}$ to $\mathcal{H}$ is orthogonal to any tangent vector to $\mathcal{N}$, so $l \in T_{p}^{\perp} \mathcal{N}$. Because of Lorentzian signature, $T_{p}^{\perp} \mathcal{N}$ has 2 independent null directions, which can be seen as 2 intersections of the null cone at $\mathrm{p}$ with 2-plane $T_{p}^{\perp} \mathcal{N}$. Let denote by $\boldsymbol{k}$, a future directed null vector which is in null direction of $T_{p}^{\perp} \mathcal{N}$ but not along $\boldsymbol{l}$. We can always do a rescaling such that we can always make $\boldsymbol{k}$ to satisfy condition $\boldsymbol{k} \cdot \boldsymbol{l}=-1$. Given $\boldsymbol{l}$ and $\mathcal{N}$ with the last condition determines the null vector $\boldsymbol{k}$ uniquely. And since $\boldsymbol{l}, \boldsymbol{k}$ are linearly independent therefore we can write $T_{p}^{\perp} \mathcal{N}=\operatorname{span}(\boldsymbol{l}, \boldsymbol{k})$.

Having a priori definition of $\mathrm{q}$, defined on $T_{p} \mathcal{N}$, using orthogonal decomposition, we can extend it to all vectors of $T_{p} \mathcal{M}$ by requiring

$$
\forall \boldsymbol{v} \in T_{p}^{\perp} \mathcal{N}, \underline{\mathrm{q}}(\boldsymbol{v}, .)=0
$$

Therefore, for any two vector $(\boldsymbol{u}, \boldsymbol{v}) \in T_{p} \mathcal{M} \times T_{p} \mathcal{M}$ we can write

$$
\begin{aligned}
& \boldsymbol{u}=\boldsymbol{u}^{\|}+\boldsymbol{u}^{\perp} \\
& \boldsymbol{v}=\boldsymbol{v}^{\|}+\boldsymbol{v}^{\perp}
\end{aligned}
$$

where $\boldsymbol{u}^{\|}, \boldsymbol{v}^{\|} \in T_{p} \mathcal{N}$ and $\boldsymbol{u}^{\perp}, \boldsymbol{v}^{\perp} \in T_{p}^{\perp} \mathcal{N}$. Using the bilinearity and the requirement in (14), we can write that

$$
\forall(\boldsymbol{u}, \boldsymbol{v}) \in T_{p} \mathcal{M} \times T_{p} \mathcal{M}, \underline{\mathrm{q}}(\boldsymbol{u}, \boldsymbol{v})=q\left(\boldsymbol{u}^{\|}, \boldsymbol{v}^{\|}\right)
$$

This is equivalent to express $\underline{\mathrm{q}}$ as

$$
\begin{aligned}
& \underline{\mathrm{q}}=\underline{\mathrm{g}}+\underline{1} \otimes \underline{\mathrm{k}}+\underline{\mathrm{k}} \otimes \underline{1} \\
& q_{\alpha \beta}=g_{\alpha \beta}+k_{\alpha} l_{\beta}+l_{\alpha} k_{\beta}
\end{aligned}
$$

\subsection{Expansion along the Null Normal}

The expansion of the cross-section $\mathcal{N}$ along the vector field $\boldsymbol{l}$ (which is null 
normal to $\mathcal{H}$ ) as follows. Given an infinitesimal small parameter $\epsilon \geq 0$, take a point $p \in \mathcal{N}$ and displace it by infinitesimal vector $\epsilon \boldsymbol{l}$ to get a new point $p_{\epsilon}$ which is close to p. Since $\boldsymbol{l}$ is also tangent to $\mathcal{H}$ and $p \in \mathcal{H}$, we have $p_{\epsilon} \in \mathcal{H}$. By repeating same process for each point on $\mathcal{N}$, keeping the value of $\epsilon$ fixed, we define a new codimension-2 surface denoted by $\mathcal{N}_{\epsilon}$. And we call this process of getting new surface as Lie-dragging along $l$ by parameter $\epsilon$.

Note that since $p_{\epsilon} \in \mathcal{H}$ for every $p \in \mathcal{N}$ therefore $\mathcal{N}_{\epsilon} \subset \mathcal{H}$. And because null direction $\boldsymbol{l}$ is transverse to $\mathcal{N}_{\epsilon}$ by construction, it follows that $\mathcal{N}_{\epsilon}$ is spacelike.

At each point $p \in \mathcal{N}$, the expansion of $\mathcal{N}$ along $\boldsymbol{l}$ is defined from the rate of change $\left(\theta_{(l)}\right)$ of the area $\delta A$ of an element of surface $\delta S$ of $\mathcal{N}$ around $\mathrm{p}$ :

$$
\theta_{(l)}=\lim _{\epsilon \rightarrow 0} \frac{1}{\epsilon} \frac{\delta A_{\epsilon}-\delta A}{\delta A}
$$

In the above formula $\delta A_{\epsilon}$ stands for the area of the surface element $\delta S_{\epsilon} \subset \mathcal{N}_{\epsilon}$ that is obtained from $\delta S$ by Lie-dragging along $\boldsymbol{l}$ by the parameter $\epsilon$.

Let us consider in some neighborhood of $\mathcal{N}$ a coordinate system

$$
x^{\alpha}=\left(\varepsilon, u, x^{2}, \cdots, x^{n-1}\right)
$$

that is adapted to $\mathcal{N}$ and $\boldsymbol{l}$ is defined as

$$
\boldsymbol{l}=\frac{\partial}{\partial \varepsilon}
$$

and the points on $\mathcal{N}$ are defined by $(\varepsilon, u)=(0,0)$. Then according to the definition of Lie-dragging we will have

$$
\mathcal{N}_{\varepsilon}=\left\{p \in \mathcal{M},\left(x^{0}(p), x^{1}(p)\right)=(\varepsilon, 0)\right\}
$$

and $\left(x^{2}, \cdots, x^{n-1}\right)$ can be viewed as coordinate system on each such $\mathcal{N}_{\epsilon}$.

Therefore area $\delta A$ of element $\delta S$ becomes

$$
\delta A=\sqrt{q} \mathrm{~d} x^{2} \cdots \mathrm{d} x^{n-1}
$$

According to the definition of Lie-dragging, the surface element $\delta S_{\varepsilon}$ on $\mathcal{N}_{\varepsilon}$ is defined by the same values of coordinates $\left(x^{2}, \cdots, x^{n-1}\right)$ as $\delta S$. In particular, the small coordinate increaments $\mathrm{d} x^{2}, \cdots, \mathrm{d} x^{n-1}$ take the same values as on $\mathcal{N}$. Therefore, the area of $\delta S_{\varepsilon}$ is

$$
\delta A_{\varepsilon}=\sqrt{q(\varepsilon)} \mathrm{d} x^{2} \cdots \mathrm{d} x^{n-1}
$$

where $q(\varepsilon)$ stands for the determinant of the components of the metric $q(\varepsilon)$ induced on $\mathcal{N}_{\varepsilon}$. And since $\mathcal{N}_{\varepsilon}$ is spacelike $q(\varepsilon)$ is positive definite.

And therefore, according to the definition of the expansion, we can write

$$
\theta_{(l)}=\lim _{\varepsilon \rightarrow 0} \frac{1}{\varepsilon} \frac{\sqrt{q(\varepsilon)}-\sqrt{q(0)}}{\sqrt{q(0)}}=\frac{1}{2} \frac{\mathrm{d}}{\mathrm{d} \varepsilon} \ln q=\frac{1}{2} \mathcal{L}_{l} \ln q
$$

Using the general law of variation of a determinant we can write 


$$
\theta_{(l)}=\frac{1}{2} \operatorname{tr}\left(Q^{-1} \mathcal{L}_{l} Q\right)
$$

where $Q$ is the matrix representing the components of $\mathrm{q}$ w.r.t coordinates $\left(x^{2}, \cdots, x^{n-1}\right)$. In index notation, we have $Q_{a b}=q_{a b}$ and $\left(\bar{Q}^{-1}\right)_{a b}=q^{a b}$. Hence, we can write

$$
\theta_{(l)}=\frac{1}{2} q^{a b} \mathcal{L}_{l} q_{a b}
$$

There is a good discussion in [10] about the Lie-derivative of metric $\underline{\mathrm{q}}$ along $\boldsymbol{l}$ and Lie-derivative of its extension also denoted by $\underline{\mathrm{q}}$ along $\boldsymbol{l}$. Without going into details from now onwards we take the definition which is extended to $T \mathcal{M}$ (tangent space) mentioned in Equation (17) which is identified with orthogonal projector onto $\mathcal{N}_{\epsilon}$ denoted by $\boldsymbol{q}$ (in short tensorial notation) which is index notation can be written as $q_{\alpha}^{\mu}$.

Now let's substitute the Equation (17) into the definition of $\theta_{(l)}$ which will give

$$
\begin{aligned}
\theta_{(l)} & =\frac{1}{2} q^{\mu v}\left(\mathcal{L}_{l} g_{\mu v}+\mathcal{L}_{l} l_{\mu} k_{v}+l_{\mu} \mathcal{L}_{l} k_{v}+\mathcal{L}_{l} k_{\mu} l_{v}+k_{\mu} \mathcal{L}_{l} l_{v}\right) \\
& =\frac{1}{2} q^{\mu v}\left(\nabla_{\mu} l_{v}+\nabla_{v} l_{\mu}\right)=q^{\mu \nu} \nabla_{\mu} l_{v}
\end{aligned}
$$

where we have used the definition of orthogonal projection

$$
q^{\mu v} l_{v}=0=q^{\mu v} k_{v}
$$

and $\mathcal{L}_{l} g_{\mu \nu}=\nabla_{\mu} l_{v}+\nabla_{v} l_{\mu}$. Note that we also used the fact that $q^{\mu v}$ is symmetric in both index.

We can go further and simplify it in following manner

$$
\begin{aligned}
\theta_{(l)} & =\left(g^{\mu v}+l^{\mu} k^{v}+k^{\mu} l^{v}\right) \nabla_{\mu} l_{v} \\
& =\nabla_{\mu} l^{\mu}+k^{v} l^{\mu} \nabla_{\mu} l_{v}+k^{\mu} l^{v} \nabla_{\mu} l_{v} \\
& =\nabla_{\mu} l^{\mu}+k^{v} \kappa l_{v}=\nabla_{\mu} l^{\mu}-\kappa \\
\Rightarrow & \theta_{(l)}=\nabla \cdot \boldsymbol{l}-\kappa
\end{aligned}
$$

We note that r.h.s of above equation is independent of choice of any particular cross-section and clearly both $\nabla \cdot \boldsymbol{l}, \kappa$ depends only on the null normal $\boldsymbol{l}$ of $\mathcal{H}$. This justifies our notation that $\theta_{(l)}$ does not refer to any $\mathcal{N}_{\varepsilon}$.

One can easily check that under rescaling $\boldsymbol{l} \mapsto \alpha \boldsymbol{l}, \theta_{(l)} \mapsto \alpha \theta_{(l)}$.

\subsection{Deformation Rate and Shear Tensor}

Let us consider a cross-section $\mathcal{N}$ of the null hypersurface $\mathcal{H}$. The deformation rate $\Theta$ of $\mathcal{N}$ is defined from the Lie derivative of the induced metric q of $\mathcal{N}$ along $\boldsymbol{l}$

$$
\Theta=\frac{1}{2} q^{*} \mathcal{L}_{l} \underline{q}
$$

where $\boldsymbol{q}^{*}$ stands for the action of the orthogonal projector $\boldsymbol{q}$ onto $\mathcal{N}$ on the bilinear form $\mathcal{L}_{l} \underline{\mathrm{q}}$. This action extends $\mathcal{L}_{l} \underline{\mathrm{q}}$, which is defined a priori on 
vectors of $T_{p} \mathcal{N}$ to all vectors of $T_{p} \mathcal{M}$, for any $p \in \mathcal{N}$, via

$$
\forall(\boldsymbol{u}, \boldsymbol{v}) \in T_{p} \mathcal{M} \times T_{p} \mathcal{M} ; \boldsymbol{q}^{*} \mathcal{L}_{l} \underline{\mathrm{q}}(\boldsymbol{u}, \boldsymbol{v})=\mathcal{L}_{l} \underline{\mathrm{q}}(\boldsymbol{q}(\boldsymbol{u}), \boldsymbol{q}(\boldsymbol{v}))
$$

Accordingly, the index notation version of Equation (30) is

$$
\Theta_{\alpha \beta}=\frac{1}{2} q_{\alpha}^{\mu} q_{\beta}^{v} \mathcal{L}_{l} q_{\mu \nu}
$$

Note since $\underline{q}$ is symmetric in indices, therefore $\Theta$ is a symmetric bilinear form. After expressing Lie-derivatives in terms of covariant derivative $\nabla$, we get

$$
\Theta_{\alpha \beta}=q_{\alpha}^{\mu} q_{\beta}^{v} \nabla_{\mu} l_{v}
$$

where we have used different orthogonality relations. We can further simplify this by writing the projector $\boldsymbol{q}$ explicitly

$$
\begin{aligned}
& \Theta_{\alpha \beta}=\left(\delta_{\alpha}^{\mu}+l^{\mu} k_{\alpha}+k^{\mu} l_{\alpha}\right)\left(\delta_{\beta}^{v}+l^{v} k_{\beta}+k^{v} l_{\beta}\right) \nabla_{\mu} l_{v} \\
& \Rightarrow \nabla_{\alpha} l_{\beta}=\Theta_{\alpha \beta}+\omega_{\alpha} l_{\beta}-l_{\alpha} k^{\mu} \nabla_{\mu} l_{\beta} \\
& \omega_{\alpha}=-k^{\mu} \nabla_{\alpha} l_{\mu}-k^{\mu} k^{\nu} \nabla_{\mu} l_{v} l_{\alpha}
\end{aligned}
$$

where we have used the fact that $\nabla_{\boldsymbol{l}} \boldsymbol{l}=\boldsymbol{\kappa} \boldsymbol{l}$. The 1 -form $\underline{\omega}$ is sometimes called the rotation 1-form of the cross-section $\mathcal{N}$.

By comparing Equation (26) an Equation (30), we notice that trace of $\underline{\Theta}$ is nothing but the expansion $\theta_{(l)}$ :

$$
\theta_{(l)}=g^{\mu v} \Theta_{\mu v}=q^{\mu v} \Theta_{\mu v}=\Theta_{\mu}^{\mu}
$$

The trace-free part of the $\underline{\Theta}$ is called the shear tensor of $\mathcal{N}$

$$
\underline{\sigma}=\underline{\Theta}-\frac{1}{n-2} \theta_{(l)} \underline{q}
$$

or in index notation

$$
\sigma_{\alpha \beta}=\Theta_{\alpha \beta}-\frac{1}{n-2} \theta_{(l)} q_{\alpha \beta}
$$

Note that by definition $\underline{\Theta}, \underline{\sigma}$ are tensor fields tangent to $\mathcal{N}$, in the sense that

$$
\forall v \in T_{p}^{\perp} \mathcal{N}, \underline{\Theta}(v, .)=0=\underline{\sigma}(v, .)
$$

One can check above using Equation (33).

Note that contrary to $\theta_{(l)}$, which depends only on $\boldsymbol{l}$ the tensor fields $\underline{\Theta}$ and $\underline{\sigma}$ depend on the specific choice of the cross-section $\mathcal{N}$, in addition to $l$.

\subsection{Null Raychaudhuri Equation}

Next the natural thing to do is to derive an evolution equation for the expansion $\theta_{(l)}$ along the null generators of $\mathcal{H}$, i.e. to evaluate the quantity $\nabla_{l} \theta_{(l)}$, where $\boldsymbol{l}$ is by hypothesis is future directed.

We start from the definition of Ricci-tensor 


$$
\nabla_{\mu} \nabla_{\alpha} l^{\mu}-\nabla_{\alpha} \nabla_{\mu} l^{\mu}=R_{\mu \alpha} l^{\mu}
$$

Now we do substitution which will give following set of equations

$$
\begin{aligned}
& \nabla_{\mu}\left(\Theta_{\alpha}^{\mu}+\omega_{\alpha} l^{\mu}-l_{\alpha} k^{v} \nabla_{v} l^{\mu}\right)-\nabla_{\alpha}\left(\theta_{(l)}+\kappa\right)=R_{\mu \alpha} l^{\mu} \\
& \Rightarrow \nabla_{\mu} \Theta_{\alpha}^{\mu}+l^{\mu} \nabla_{\mu} \omega_{\alpha}-\nabla_{\alpha}\left(\theta_{(l)}+\kappa\right)+\left(\theta_{(l)}+\kappa\right) \omega_{\alpha} \\
& \quad-\Theta_{\alpha \mu} k^{v} \nabla_{v} l^{\mu}-\left(\omega_{\mu} k^{v} \nabla_{v} l^{\mu}+\nabla_{\mu} k^{v} \nabla_{v} l^{\mu}+k^{v} \nabla_{\mu} \nabla_{v} l^{\mu}\right) l_{\alpha}=R_{\mu \alpha} l^{\mu}
\end{aligned}
$$

Now we contract above equation with $l^{\alpha}$

$$
l^{v} \nabla_{\mu} \Theta_{v}^{\mu}+l^{v} l^{\mu} \nabla_{\mu} \omega_{v}-l^{\mu} \nabla_{\mu}\left(\theta_{(l)}+\kappa\right)+\left(\theta_{(l)}+\kappa\right) \omega_{\mu} l^{\mu}=R_{\mu v} l^{\mu} l^{v}
$$

On can further simplify the first two terms on 1.h.s and get following expression

$$
-\Theta^{\mu v} \Theta_{\mu v}+l^{\mu} \nabla_{\mu}\left(\omega_{v} l^{v}\right)-l^{\mu} \nabla_{\mu}\left(\theta_{(l)}+\kappa\right)+\theta_{(l)} \omega_{\mu} l^{\mu}=R_{\mu v} l^{\mu} l^{v}
$$

Now note that

$$
l^{\alpha} \omega_{\alpha}=-k^{\mu} l^{\alpha} \nabla_{\alpha} l_{\mu}-k^{\mu} k^{\nu} \nabla_{\mu} l_{v} l_{\alpha} l^{\alpha}=-k^{\mu} \kappa l_{\alpha}=\kappa
$$

Therefore, Equation (42) implies following expression

$$
-\Theta^{\mu v} \Theta_{\mu v}-l^{\mu} \nabla_{\mu} \theta_{(l)}+\kappa \theta_{(l)}=R_{\mu v} l^{\mu} l^{v}
$$

One can further simplify the firt term in 1.h.s to get

$$
\Theta^{\mu v} \Theta_{\mu v}=\sigma^{\mu v} \sigma_{\mu v}+\frac{1}{n-2} \theta_{(l)}^{2}=\sigma^{a b} \sigma_{a b}+\frac{1}{n-2} \theta_{(l)}^{2}
$$

Hence, we can write

$$
\nabla_{l} \theta_{(l)}=\kappa \theta_{(l)}-\sigma^{\mu v} \sigma_{\mu v}-\frac{1}{n-2} \theta_{(l)}^{2}-\underline{R}(\boldsymbol{l}, \boldsymbol{l})
$$

The above equation is known as Raychaudhuri equation.

If the spacetime $(\mathcal{M}, \underline{g})$ is ruled by General Relativity, i.e. if $\underline{g}$ obeys Einstein equation, we can write

$$
\underline{R}(\boldsymbol{l}, \boldsymbol{l})=\frac{2}{n-2} \Lambda \underline{g}(\boldsymbol{l}, \boldsymbol{l})+8 \pi\left[\underline{T}(\boldsymbol{l}, \boldsymbol{l})-\frac{1}{n-2} T \underline{g}(\boldsymbol{l}, \boldsymbol{l})\right]=8 \pi \underline{T}(\boldsymbol{l}, \boldsymbol{l})
$$

where $\Lambda$ is cosmological constant.

Then null Raychaudhuri equation becomes

$$
\nabla_{l} \theta_{(l)}=\kappa \theta_{(l)}-\sigma^{\mu v} \sigma_{\mu v}-\frac{1}{n-2} \theta_{(l)}^{2}-8 \pi \underline{T}(\boldsymbol{l}, \boldsymbol{l})
$$

\subsection{Killing Horizons}

A Killing horizon is a null hypersurface $\mathcal{H}$ in a spacetime $(\mathcal{M}, \underline{g})$ admitting a Killing vector field $\boldsymbol{\xi}$ such that, on $\mathcal{H}, \boldsymbol{\xi}$ is normal to $\mathcal{H}$.

From the above definition it is clear that Killing horizon requires that spacetime $(\mathcal{M}, \underline{g})$ has some continuous symmetry. And a definition equivalent to above one is following:

A Killing horizon is a null hypersurface $\mathcal{H}$ whose null geodesic generators 
are orbits of a one-parameter group of isometries of $(\mathcal{M}, \underline{g})$.

The above definition also implies that Killing vector field $\boldsymbol{\xi}$ is null $\left.\xi \cdot \xi\right|_{\mathcal{H}}=0$ and it is non-vanishing on $\mathcal{H},\left.\boldsymbol{\xi}\right|_{\mathcal{H}} \neq 0$.

Let $\mathcal{H}$ be a Killing horizon with cross-sections that are closed manifolds. And let us select null normal $\boldsymbol{l}$ that coincides with the Killing vector on $\mathcal{H}$ : $\boldsymbol{l} \stackrel{\mathcal{H}}{\boldsymbol{\xi}}$ then one can easily show that the expansion rate tensor $\underline{\Theta}$ vanishes identically.

Let $\kappa$ be the non-affinity coefficient of the bull normal $\boldsymbol{l}$ coinciding with the Killing vector $\boldsymbol{\xi}$ on a Killing horizon $\mathcal{H}$. Then we can write $\nabla_{\xi} \xi^{\mathcal{H}}=\kappa \xi$. Using the Killing equation one can show that

$$
\begin{aligned}
& \nabla_{\alpha}\left(\xi_{\mu} \xi^{\mu}\right) \stackrel{\mathcal{H}}{=}-2 \kappa \xi_{\alpha} \\
& d(\xi \cdot \xi) \stackrel{\mathcal{H}}{=}-2 \kappa \xi
\end{aligned}
$$

Another interesting relation one can find using Frobenius identity, which is $\kappa^{2} \stackrel{\mathcal{H}}{=}-2 \nabla_{\mu} \xi^{v} \nabla^{\mu} \xi^{v}$.

\section{Laws of Black Hole Mechanics}

\subsection{The Zeroth Law of Black Hole Mechanics}

We are now ready to establish a result of great importance in black hole physics which states that non-affinity coefficient $\kappa$ defined earlier is constant on a Killing horizon, provided some mild energy condition holds.

Let us denote by $\boldsymbol{l}$ the null normal to $\mathcal{H}$ that coincides with the Killing vector field $\boldsymbol{l}^{\mathcal{H}} \boldsymbol{\xi}$. The vector field $\boldsymbol{l}$ is then a symmetry generator on $\mathcal{H}$, which implies

$$
\mathcal{L}_{l} \kappa=0
$$

which means that $\kappa$ is constant along the field lines of $\boldsymbol{l}$. Now the only thing that remains to show is that $\kappa$ also does not vary from one field line to another field line.

To show that, let us consider a cross-section $\mathcal{N}$ of $\mathcal{H}$ and project the contracted Ricci identity in Equation (40) onto it via the orthogonal projector $\boldsymbol{q}$ introduced earlier

$$
\begin{aligned}
& \nabla_{\mu} \Theta_{\nu}^{\mu} q_{\alpha}^{v}+l^{\mu} \nabla_{\mu} \omega_{\nu} q_{\alpha}^{v}-\nabla_{v}\left(\theta_{(l)}+\kappa\right) q_{\alpha}^{\nu} \\
& +\left(\theta_{(l)}+\kappa\right) \omega_{\nu} q_{\alpha}^{\nu}-\Theta_{\alpha \mu} k^{\nu} \nabla_{\nu} l^{\mu}=R_{\mu \nu} l^{\mu} q_{\alpha}^{v}
\end{aligned}
$$

Now using the properties of the projector and the fact that $\mathcal{H}$ is a non-expanding horizon which means we can set $\underline{\Theta}=0$ and $\theta_{(l)}=0$, the above equation reduces to

$$
l^{\mu} \nabla_{\mu} \omega_{v} q_{\alpha}^{v}-\nabla_{v} \kappa q_{\alpha}^{v}+\kappa \omega_{v} q_{\alpha}^{v}=R_{\mu \nu} l^{\mu} q_{\alpha}^{v}
$$

Using the definition of Lie-derivative we can write $\mathcal{L}_{l} \omega_{v}=l^{\mu} \nabla_{\mu} \omega_{v}+\omega_{\mu} \nabla_{v} l^{\mu}$ but since $\boldsymbol{l}$ is a symmetry generator of $\mathcal{H}$, we have $\mathcal{L}_{l} \underline{\omega} \stackrel{\mathcal{H}}{=} 0$, which implies that $l^{\mu} \nabla_{\mu} \omega_{v} \stackrel{\mathcal{H}}{=}-\omega_{\mu} \nabla_{v} l^{\mu}$. Using this property and the Equation (34), we can 
simplify the above equation in following form

$$
-\nabla_{v} \kappa q_{\alpha}^{v}=R_{\mu v} l^{\mu} q_{\alpha}^{v}
$$

In the above equation appears the covariant derivative of $\kappa$ along $\mathcal{N}$, which we denoted by ${ }^{\mathcal{N}} \mathcal{D}$ and defined by ${ }^{\mathcal{N}} \mathcal{D} \equiv \nabla_{v} \kappa q_{\alpha}^{v}$.

Using Einstein equation

$$
\begin{aligned}
& { }^{\mathcal{N}} \mathcal{D}_{\alpha} \kappa=-\frac{2}{n-2} \Lambda \underbrace{g_{\mu \nu} l^{\mu} q_{\alpha}^{v}}_{=0}-8 \pi(T_{\mu \nu} l^{\mu} q_{\alpha}^{\nu}-\frac{1}{n-2} T \underbrace{g_{\mu \nu} l^{\mu} q_{\alpha}^{\nu}}_{=0}) \\
& \Rightarrow{ }^{\mathcal{N}} \mathcal{D} \kappa=-8 \pi T_{\mu \nu} l^{\mu} q_{\alpha}^{\nu}
\end{aligned}
$$

We shall assume that matter obey null dominant energy condition:

$$
\begin{aligned}
& \underline{W}=-\underline{T}(\boldsymbol{l}, .) \\
& W^{\alpha}=-g^{\alpha \nu} T_{\mu \nu} l^{\mu}=-T_{\mu}^{\alpha} l^{\mu}
\end{aligned}
$$

is future directed null or time-like for any future directed null vector $\boldsymbol{l}$.

Note that null dominant energy condition implies the null energy condition since

$$
\underline{T}(\boldsymbol{l}, \boldsymbol{l})=-\boldsymbol{W} \cdot \boldsymbol{l} \geq 0
$$

this inequality holds because both $W$ and $\boldsymbol{l}$ are future directed.

Now note that on r.h.s of Equation (54) we have orthogonal projection of $W$ onto $\mathcal{N}$. If we assume null dominant energy condition, the null energy condition holds, and we have according to Equation (48) for non-expanding horizon $\underline{T}(\boldsymbol{l}, \boldsymbol{l})=0=\boldsymbol{W} \cdot \boldsymbol{l}$. This implies that $\boldsymbol{W}$ is tangent to $\mathcal{H}$ and since $\mathcal{H}$ is a null-hyperrsurface $W$ is either null or spacelike vector. Now according to null dominant energy condition $W$ can't be spacelike which implies $W$ is null-like and it is collinear to $\boldsymbol{l}$. Therefore, $q_{v}^{\alpha} W^{v}=0$ and which implies

$$
{ }^{\mathcal{N}} \mathcal{D} \kappa=0
$$

which shows $\kappa$ is constant over $\mathcal{N}$. Therefore, we are able to show that $\kappa$ is indeed a constant on the horizon $\mathcal{H}$.

\subsection{The First Law of Black Hole Mechanics}

The event horizon area is related to properties of a stationary black hole which are like its mass, angular momentum and surface gravity. First law of black hole exactly gives us a simple equation which governs how a small change in one of the above properties will influence others once the black hole reach to equilibrium state.

Under a small amount of perturbation in terms of matter, the local value of stress-energy tensor $T_{\mu \nu}$ near black hole horizon will change slightly by an amount denoted by $\delta T_{\mu \nu}$. The resulting change in black hole area can be calculated from the null Raychaudhuri equation. The change in $\theta_{(l)}^{2}, \sigma_{a b} \sigma^{a b}$ will only come from changes in local curvature through Einstein's equation which can be neglected compare to $\delta T_{\mu \nu}$ and so the Raychaudhuri equation simplifies to 


$$
\nabla_{l} \theta_{(l)}=\frac{\mathrm{d} \theta}{\mathrm{d} \lambda}=-8 \pi \delta T_{\mu \nu} l^{\mu} l^{v}
$$

where $\lambda$ is an affine parameter. Another property of $\kappa$ that will be stated without proof [5] is that it represents a proportionality factor between the affinely parametrized null geodesics that generate the event horizon and the Killing vector field parametrization. If we label the affine parameter $\lambda$, then each component of the affinely parametrized null geodesic generators $l$ a and the Killing vector $\chi$ is

$$
\lambda \boldsymbol{l}=\frac{1}{\kappa} \chi
$$

The most general black hole solution to the Einstein equation contains a black hole orthogonal to a Killing vector field composed of a timelike and a periodic spacelike part. In this case, the black hole event horizon is a null hypersurface orthogonal to a linear combination of two Killing vectors written as [13]

$$
\chi^{a}=\xi^{a}+\Omega \phi^{a} l^{a}=\frac{1}{\kappa \lambda}\left(\xi^{a}+\Omega \phi^{a}\right)
$$

To get the effect of perturbation $\delta T_{\mu \nu}$ on the black hole once it reaches in equilibrium, we need too integrate both side of Raychaudhuri equation over the event horizon surface and over all future values of $\lambda$

$$
\begin{aligned}
& \kappa \int \mathrm{d}^{2} S \int_{0}^{\infty} \lambda \frac{\mathrm{d} \theta}{\mathrm{d} \lambda}=-8 \pi\left[\int_{0}^{\infty} \mathrm{d} \lambda \int \mathrm{d}^{2} S \delta T_{\mu \nu} \xi^{\mu} l^{\nu}+\Omega \int_{0}^{\infty} \mathrm{d} \lambda \int \mathrm{d}^{2} S \delta T_{\mu \nu} \phi^{\mu} l^{\nu}\right] \\
& \Rightarrow \kappa \int \mathrm{d}^{2} S \int_{0}^{\infty} \theta \mathrm{d} \lambda=8 \pi\left[\int_{0}^{\infty} \mathrm{d} \lambda \int \mathrm{d}^{2} S \delta T_{\mu \nu} \xi^{\mu} l^{\nu}+\Omega \int_{0}^{\infty} \mathrm{d} \lambda \int \mathrm{d}^{2} S \delta T_{\mu \nu} \phi^{\mu} l^{\nu}\right] \\
& \Rightarrow \kappa \delta A=8 \pi(\delta M-\Omega \delta J)
\end{aligned}
$$

where from to go second line from first line we have used integration by parts and throw away the boundary term. To get third line from second line, we have used the fact that l.h.s is nothing but the integral of the expansion of each infinitesimal area element of event horizon over the surface of event horizon which is nothing but the infinitesimal change in event horizon surface $\delta A$ (according to the definition of $\theta_{(l)}$ ) caused by $\delta T_{\mu \nu}$.

On the r.h.s we have action of vector fields on $\delta T_{\mu \nu}$ is simply project onto one of its complonents. Since $\vec{\xi}, \vec{k}$ both are future directed in time the first integral will be an integral of the $T_{00}$ component, which is for an asymptotic observer nothing but the change in mass $\delta M$ of the system. The $\delta T_{\mu \nu} \phi^{\mu} l^{v}$ is a projection onto the time- $\phi$ component of $\delta T_{\mu \nu}$, which is just the negative of angular momentum $J$ for an asymptotic observer.

Note that first law also depends on zeroth law in a sense because we have used the fact that $\kappa$ is constant to do the integral.

\subsection{The Area Increase Theorem or The Second Law of Black Hole Mechanics}

Another important geometric quantity is the area of the event horizon which we have not discussed yet. This theorem states that the area of a black hole event 
horizon as viewed by a distant observer must never decrease with time. If we assume null-energy condition and since $\sigma_{a b} \sigma^{a b}$ is positive definite we can write (in 4-dimension with affine parametrization and for geodesic congruences mentioned in [14] where basically first term in Equation (48) got removed)

$$
\begin{aligned}
& \frac{\mathrm{d} \theta_{(l)}}{\mathrm{d} \lambda}=-\frac{1}{2} \theta_{(l)}^{2}-T_{\mu \nu} l^{\mu} l^{v}-\sigma_{a b} \sigma^{a b} \\
& \Rightarrow \frac{\mathrm{d} \theta_{(l)}}{\mathrm{d} \lambda} \leq-\frac{1}{2} \theta_{(l)}^{2}
\end{aligned}
$$

which we can write in following ways(we just omit $l$ from subscript)

$$
\begin{aligned}
& \frac{\mathrm{d} \theta}{\mathrm{d} \lambda} \leq-\frac{\theta^{2}}{2} \\
& \int_{\theta_{0}}^{\theta} \frac{\mathrm{d} \theta}{\theta^{2}} \leq-\int_{0}^{\lambda} \frac{1}{2} \mathrm{~d} \lambda \\
& \frac{1}{\theta(\lambda)} \geq \frac{1}{\theta_{0}}+\frac{1}{2} \lambda
\end{aligned}
$$

Note that if $\theta_{0}<0$ then there exists a value of $\lambda$ for which r.h.s of above equation vanishes and therefore $\theta_{(l)}(\lambda)$ at that particluar value of $\lambda$ diverges which is unphysical therefore $\theta_{0}$ must be positive definite $\theta_{0} \geq 0$ which means that $\theta(\lambda)$ is always positive which shows that area of event horizon never decreases under time evolution according to the definition Equation (18).

\subsection{The Third Law of Black Hole Mechanics}

Third law states that surface gravity defined earlier is positive definite that is $\kappa \geq 0$ which comes from the fact that if $\kappa<0$ then black hole seem repulsive from distant observer, going against all geometric property of metric of black holes have.

This law can also be proven by calculating the value of $\kappa$ for most general situation which is the case for stationary black hole metric, the Kerr metric. The non-negativity property of $\kappa$ is guranteed by the physical dmand that the solution does not have any closed timelike curves [5].

As we have told earlier laws of black hole mechanics strongly depend on null-energy condition and people consider these laws seriously often without being bothered by the strong assumption of null energy condition behind it. Now we will look at why often people do think that null energy condition might be guranteed always. First we will look at the classical matter description of it.

\section{Null Energy Condition}

\subsection{Perfect Fluid Description}

When we thin of classical matters the first thing that comes to our mind is a perfect fluid system which is most often taken in General Relativity to describe the matter that governs the geometry of spacetime. Stress-energy tensor of perfect fluid system is given by 


$$
T_{\mu v}=(\rho+P) u_{\mu} u_{v}+P g_{\mu v}
$$

where $\rho$ is the matter energy density and $P$ is the isotropic pressure exerted by the system. Let's take a arbitrary null vector denoted by $n^{\mu}$ which by definition satisfies $n_{\mu} n^{\mu}=0$. Therefore

$$
T_{\mu \nu} u^{\mu} u^{v}=(\rho+P)\left(u_{\mu} n^{\mu}\right)^{2}+P g_{\mu \nu} n^{\mu} n^{v}=(\rho+P)\left(u_{\mu} n^{\mu}\right)^{2}
$$

Since, most of the time we physically admit that $\rho \geq 0$ and $P \geq 0$ which implies from the above equation that

$$
T_{\mu v} u^{\mu} u^{v} \geq 0
$$

for perfect fluid which means perfect fluid satisfies null energy condition. (But $P \geq 0$ may not be the case always because pressure can be thought as the response of the system under compression or expansion of the system size in terms of change in energy of the system according to first law of thermodynamics and therefore it can be negative too. There is absolutely no problem in having negative pressure just like negative heat capacity is also not problematic at all.)

\subsection{Minimally Coupled Real Scalar Field Theory}

Now let's consider a minimally coupled real scalar field theory which is also often treated as a matter in different context of comology and in other subbranches of General Relativity.

Action of such system is given by

$$
\mathcal{A}=-\int \sqrt{-g} \mathrm{~d}^{4} x\left[\frac{1}{2} g^{\mu \nu} \partial_{\mu} \phi \partial_{\nu} \phi+\frac{1}{2} m^{2} \phi^{2}+U(\phi)\right]
$$

where $g_{\mu \nu}$ is the metric of the background classical geometry and $U(\phi)$ is an arbitrary self interacting potential. And the stress-energy tensor of this system is given by as follows

$$
\begin{aligned}
T_{\mu \nu}(x)=- & \frac{2}{\sqrt{-g}} \frac{\delta \mathcal{A}}{\delta g^{\mu \nu}(x)} \\
\Rightarrow T_{\mu \nu}(x)= & \partial_{\mu} \phi(x) \partial_{\nu} \phi(x)-\frac{1}{2} g_{\mu \nu}(x) g^{\rho \sigma}(x) \partial_{\rho} \phi(x) \partial_{\sigma} \phi(x) \\
& \quad-\frac{1}{2} g_{\mu \nu}(x) m^{2} \phi^{2}(x)-g_{\mu \nu}(x) U(\phi)
\end{aligned}
$$

Now as earlier for an arbitrary null vector $n^{\mu}$ we will get

$$
T_{\mu \nu}(x) n^{\mu} n^{v}=\partial_{\mu} \phi(x) \partial_{\nu} \phi(x) n^{\mu} n^{v}=\left(n^{\mu} \partial_{\mu} \phi(x)\right)^{2}=\left(\nabla_{n} \phi(x)\right)^{2}
$$

Now note that since $\phi$ is a real scalar field therefore we also expect that $\nabla_{n} \phi$ is also real at any spacetime point therefore we find that $T_{\mu v}(x) n^{\mu} n^{v} \geq 0, \forall x \in \mathcal{M}$ (space time manifold) null energy condition also holds for this system.

\subsection{Minimally Coupled U(1) Gauge Field}

Next system which is often used as a matter is minimally coupled gauge fields 
which describes electromagnetism in curved spacetime. This description is required to deal with charged matter in curved spacetime for example to derive reissner nordstrom metric in General Relativity such matter has been used which describes geometry of a charged black hole.

Action is given by(without any sources or external currents)

$$
\mathcal{A}_{\mathrm{EM}}=-\frac{1}{4} \int \sqrt{-g} \mathrm{~d}^{4} x F_{\mu \nu} F_{\rho \sigma} g^{\mu \rho} g^{\nu \sigma}
$$

where field strength tensor $F_{\mu \nu}$ is defined by $F_{\mu \nu}=\nabla_{\mu} A_{v}-\nabla_{v} A_{\mu}=\partial_{\mu} A_{v}-\partial_{v} A_{\mu}$.

As ealier using the definition we will find that stress-energy tensor of this matter is following

$$
T_{\mu v}(x)=g^{\alpha \beta}(x) F_{\alpha \mu}(x) F_{\beta v}(x)-\frac{1}{4} g_{\mu v}(x) F_{\alpha \beta}(x) F^{\alpha \beta}(x)
$$

Here we will find that for any arbitrary null vector $n^{\mu}$

$$
T_{\mu \nu}(x) n^{\mu} n^{v}=g^{\alpha \beta}(x) F_{\alpha \mu}(x) F_{\beta v}(x) n^{\mu} n^{v}
$$

Let's define vector field $V_{\alpha}(x) \equiv F_{\alpha \mu}(x) n^{\mu}$ and in terms of this vector field we can write

$$
T_{\mu \nu}(x) n^{\mu} n^{v}=g^{\alpha \beta}(x) V_{\alpha}(x) V_{\beta}(x)
$$

as norm of this vector field.

Now note that $V_{\mu}(x) n^{\mu}=F_{\mu v}(x) n^{\mu} n^{v}=0$ which means that $V_{\mu}(x)$ is orthogonal to the chosen null vector and therefore this vector field $V^{\mu}(x)$ must be a spacelike or null vector field which means $T_{\mu v}(x) n^{\mu} n^{v}=g^{\alpha \beta}(x) V_{\alpha}(x) V_{\beta}(x) \geq 0, \forall x \in \mathcal{M}$. Therefore, for this matter field theory also null energy condition of stress-energy tensor holds.

\subsection{Fermionic Matter}

Action of a fermionic or spinor field theory is given by

$$
\begin{aligned}
& S=\int \sqrt{-g} \mathrm{~d}^{4} x \mathcal{L} \\
& \mathcal{L}=T+V(\psi, \bar{\psi}) \\
& T=\frac{i}{2} \bar{\psi} \vec{\nabla} \psi \\
& \nabla=\gamma^{\mu} \nabla_{\mu} \\
& \nabla_{\mu}=\partial_{\mu}+\Gamma_{\mu}
\end{aligned}
$$

where $\Gamma_{\mu}$ 's are spin connection which are derived in [15] in terms of tetrads.

Stress-energy tensor of this matter is given by as follows

$$
\begin{aligned}
T^{\mu \nu} & =-\frac{2}{\sqrt{-g}} \frac{\delta S}{\delta g_{\mu v}}=-\frac{E^{\mu c}}{2 e} \frac{\delta S}{\delta e^{c}}+(\mu \leftrightarrow v) \\
T_{\mu \nu} & =\frac{e_{c \mu}}{2 e} \frac{\delta S}{\delta E^{v d}}+(\mu \leftrightarrow v)=\frac{i}{4} \bar{\psi}\left[\gamma_{\mu} \nabla_{v}-\vec{\nabla}_{\mu} \gamma_{\nu}\right]+(\mu \leftrightarrow v)-\frac{1}{4} g_{\mu v} \mathcal{L}
\end{aligned}
$$

where $e_{v}^{c}$ and $E_{c}^{\mu}$ are the components of the transformation matrix between tetrad and coordinate(or canonical) basis. 
For an arbitrary null vector denoted by $n^{\mu}$, we will find

$$
T_{\mu \nu} n^{\mu} n^{v}=\frac{i}{2} \bar{\psi}\left[n^{\mu} \gamma_{\mu} n^{v} \nabla_{v}-n^{\mu} \overrightarrow{\nabla_{\mu}} n^{v} \gamma_{\nu}\right]
$$

Now note that

$$
\begin{aligned}
\left(n^{\mu} \gamma_{\mu}\right)^{2} & =n^{\mu} \gamma_{\mu} n^{v} \gamma_{v}=\frac{1}{2} \underbrace{n^{\mu} n^{v}\left[\gamma_{\mu}, \gamma_{v}\right]}_{=0}+\frac{1}{2} n^{\mu} n^{v}\left\{\gamma_{\mu}, \gamma_{v}\right\} \\
& =\frac{1}{2} n^{\mu} n^{v} \times 2 g_{\mu v}=0 \\
\Rightarrow n^{\mu} \gamma_{\mu} & =0
\end{aligned}
$$

Therefore, we found that $T_{\mu v}(x) n^{\mu} n^{v}=0, \forall x \in \mathcal{M}$ which means for this case also null energy condition is satisfied by the matter.

\subsection{Classical QED with Fermionic Current}

If we add to action $\mathcal{A}_{\mathrm{EM}}$ following term

$$
\mathcal{A}_{\text {source }}=-\int \sqrt{-g} \mathrm{~d}^{4} x g_{\mu v} A^{\mu} j^{v}
$$

where $j^{\mu}$ is the source or external current, then we get correction term in stress-energy tensor which is of following form

$$
T_{\mu \nu}^{(2)}(x)=j_{\mu}(x) A_{v}(x)+j_{v}(x) A_{\mu}(x)-g_{\mu \nu}(x) j^{\rho}(x) A_{\rho}(x)
$$

Note that therefore

$$
T_{\mu v}^{(2)}(x) n^{\mu} n^{v}=2\left(j_{\mu}(x) n^{\mu}\right)\left(A_{v}(x) n^{v}\right)
$$

For an arbitrary source term we can't comment on the sign of above quantity but if the source term is $j_{\mu}(x)=\bar{\psi}(x) \gamma_{\mu} \psi(x)$. then according to Equation (77), we will find that $T_{\mu v}^{(2)}(x) n^{\mu} n^{v}=0, \forall x \in \mathcal{M}$ which means atleast for this particular source matter satisfies null-energy condition but other-wise for any arbitrary current NEC might be violated.

\subsection{Few Remarks on Classical Matter}

- As we have see in last couple of subsections that most often used classical matters indeed satisfy null-energy condition. So, considering such examples one can safely apply laws of black hole mechanics in any situation.

- But there are examples even in classical matters where null-energy condition does not hold, one such example is show in last subsection and we will see few more examples of classical field theories where matter does not hold null-energy condition with out facing any unphysical problems.

- Except fermionic case if we look at the quantum version of above given field theories we will find in obvious manner that null-energy condition does not hold because there through quantization procedure fields at any spacetime point becomes operators therefore our previous arguments does not work there. Therefore, in QFT in curved spacetime theorems or laws like laws of 
black hole mechanics face serious problem.

One of the reason that people often hesitate to consider violation of null-energy condition condition because it has its own standpoint in cosmological models. So, let's go through that importance first.

Standard FLRW metric is given by

$$
\mathrm{d} s^{2}=-\mathrm{d} t^{2}+a^{2}(t)\left[\frac{\mathrm{d} r^{2}}{1-k r^{2}}+r^{2}\left(\mathrm{~d} \theta^{2}+\sin ^{2} \theta \mathrm{d} \varphi^{2}\right)\right]
$$

Only non-trivial components of Einstein equations are

$$
\begin{aligned}
& \rho=\frac{3}{8 \pi G}\left(\frac{\dot{a}^{2}}{a^{2}}+\frac{k}{a^{2}}\right) \\
& p=\frac{1}{8 \pi G}\left(\frac{2 \ddot{a}}{a}+\frac{\dot{a}^{2}}{a}+\frac{k}{a^{2}}\right)
\end{aligned}
$$

And the conservation law gives that

$$
\dot{\rho}=-3 \frac{\dot{a}}{a}(\rho+p)
$$

Holding NEC condition means that $\operatorname{sign}(\dot{\rho})=-\operatorname{sign}(\dot{a})$ which basically says that density of universe decreases as its size increases which is physically consistent. Therefore, violation of NEC demands an unphysical situation and that is also independent of whether universe is open, closed or flat.

But note that the above consequence strongly depends on the assumption that we can model the matter of entire universe as a perfect and homogeneous fluid which is a strong assumption.

\subsection{Cosmology with NEC Violation}

We now give an physically consistent toy example of a universe modelled by viscous fluid. So for mathematical convenience we choose $k=0$ (spatially flat FRW spacetime). In standard perfect fluid case as we know that

$$
\begin{aligned}
& \rho=\frac{3}{8 \pi G} H^{2} \\
& p=-\frac{1}{8 \pi G}\left(2 \dot{H}^{2}+3 H^{2}\right), H=\frac{\dot{a}}{a} \\
& \Rightarrow \omega_{\text {eff }}=\frac{p}{\rho}=-1-\frac{2 \dot{H}}{3 H^{2}}
\end{aligned}
$$

It is well-known that ordinary matter and radiation are decoupled and separately satisfy the same form of energy conservation law, but it is not necessarily true for other kind of energy. We already know Dark matter is an important component we require to describe visible universe correctly. We will consider a model where a viscous fluid and dark matter are coupled [16] [17]. Their energy conservation laws are given by(assuming $p_{D M} \approx 0$ )

$$
\begin{aligned}
& \dot{\rho}_{F}+3 H\left(\rho_{F}+p_{F}\right)=-Q \rho_{F} \\
& \dot{\rho}_{D M}+3 H \rho_{D M}=Q \rho_{F}
\end{aligned}
$$


Now for simplicity we assume here $Q$ to be a constant.

Note that from above set of equation that NEC will be violated if $\dot{\rho}_{F}+Q \rho_{F}>0$ which means $\rho_{F}>\rho_{F}^{(0)} \mathrm{e}^{-Q t}$ where $\rho_{F}^{(0)}$ is some positive constant. And $\operatorname{sign}\left(\dot{\rho}_{F}+Q \rho_{F}\right)=\operatorname{sign} \frac{\dot{a}}{a}$ if NEC is violated which means $\operatorname{sign}\left(\dot{\rho}_{F}\right)=\operatorname{sign}\left(\frac{\dot{a}}{a}-Q \rho_{F}\right)$ which could also generate negative sign for $\dot{\rho}_{F}$ even if NEC is violated.

The fluid equation of state that we consider here is of following form

$$
p_{F}=\omega\left(\rho_{F}\right) \rho_{F}-H \zeta\left(\rho_{F}\right)
$$

where $3 H$ being 4-velocity of cosmic fluid and $\omega\left(\rho_{F}\right)$ for time being an arbitrary function of $\rho_{F} \cdot \zeta\left(\rho_{F}\right)$ is the bulk viscosity which generally depends on $\rho_{F}$ which is physically reasonable. On the thermodynamical basis, in order to have positive entropy change in an irreversible process we need $\zeta\left(\rho_{F}\right)>0$. Note that such viscous fluid is a special case of more general inhomogeneous fluid introduced in [18].

Note that from Equation (85) that here because of the presence of the coupling $Q$ on r.h.s we can have NEC violataion depending on the value of coupling $Q$.

We go little more into discussion to get to know whether there is any physical restriction on the value of $Q$ or not. By taking into account Equation (86), we can rewrite first equation in Equation (85) as follows

$$
\dot{\rho}_{F}+3 H \rho_{F}\left(1+\omega\left(\rho_{F}\right)\right)+Q \rho_{F}=9 H^{2} \zeta\left(\rho_{F}\right)
$$

For simplicity let assume $\omega\left(\rho_{F}\right)=\omega$ to be some constant and the bulk viscosity is in the form $\zeta=\zeta_{0} H^{\alpha}$ where $\alpha$ is a real number(Note that from the Friedman equation we can write $H$ in terms of $\rho_{F}$ ). In this case general solution of Equation (87) is given by

$$
\rho_{F}=\rho_{0 F} \frac{\mathrm{e}^{-Q t-3 \omega \ln a(t)}}{a^{3}(t)}+\frac{9 \zeta_{0} \mathrm{e}^{-Q t-3 \omega \ln a(t)}}{a^{3}(t)} \times \int^{t} \mathrm{~d} t^{\prime} \mathrm{e}^{Q t^{\prime}+3 \omega \ln a\left(t^{\prime}\right)} a\left(t^{\prime}\right) \dot{a}^{2}\left(t^{\prime}\right) H^{\alpha}\left(t^{\prime}\right)(
$$

(mentioned in [17]) where $\rho_{0 F}$ is a positive integration constant. And note that we can write pre-factors of each term as $\frac{\mathrm{e}^{-Q t}}{a^{3(1+\omega)}}$ which would be more than $\mathrm{e}^{-Q t}$ if $\omega<-1$ which is consistent with our previous analysis(in this case because of exponential prefactor $\rho_{F}$ is decraesing as universe increases in its size which is consistent).

One important case that we may study is de Sitter solution with $h=H_{0}$ with present value of accelerated universe $H_{0} \approx 10^{-33} \mathrm{eV}$ in order to reconstruct standard cosmology. In that case we will find

$$
\rho_{F}=\rho_{0 F} \mathrm{e}^{-t\left(Q+3 H_{0}(1+\omega)\right)}+\frac{9 H_{0}^{\alpha+2} \zeta_{0}}{Q+3 H_{0}(1+\omega)}
$$

and it follows the solution of dark matter

$$
\rho_{D M}=\rho_{0 D M} \mathrm{e}^{-3 H_{0} t}-\rho_{0 F} \frac{Q}{Q+3 H_{0} \omega} \mathrm{e}^{-t\left(Q+3 H_{0}(1+\omega)\right)}+\frac{3 H_{0}^{\alpha+1} Q \zeta_{0}}{Q+3 H_{0}(1+\omega)}
$$


where $\rho_{0 D M}$ is a positive constant. And one can check easily that if $\zeta_{0} \neq 0$, then only way to satisfy Friedmann equations is to set $\rho_{0 F}=0=\rho_{0 D M}$ in which case

$$
\frac{\rho_{D M}}{\rho_{F}}=\frac{Q}{3 H_{0}} \simeq \frac{1}{3} \text { ration determined from experiments }
$$

which shows that value of $Q$ must be $Q=H_{0}$.

Using Friedmann equations one can also derive that

$$
\omega=-\frac{4}{3}+\frac{4}{3} H_{0}^{\alpha-1} \zeta_{0}(8 \pi G)
$$

which shows that $\omega=-1$ which means de-Sitter solution iff $\zeta_{0}=\frac{1}{32 \pi G H_{0}^{\alpha-1}}$.

So this is one of the simple examples where one can show violation of NEC without physical inconsistencey.

Similarly a detailed calculation how universe exits from matter dominatd era and reach de Sitter scenarion is described in [16] by considering 2 approximations $Q=H_{0} \ll H$ and $\rho_{F} \ll \rho_{D M \underline{M}}$. Accordingly modify the conservation equations with scaling factor $a(t)=a_{0} t^{\frac{2}{3}}$.

\subsection{Violation of NEC in Non-Minimal Coupled Scalar Field}

When a classical non-minimally coupled scalar field acts as a source of gravity, null energy conditions can be violated depending on the form and the value of the curvature coupling.

If we consider matter action to be of following form [19] [20]

$$
\mathcal{S}=-\int \sqrt{-g} \mathrm{~d}^{4} x\left(\frac{1}{2} g^{\mu \nu} \partial_{\mu} \phi_{\xi} \partial_{\nu} \phi_{\xi}+V\left(\phi_{\xi}\right)+\frac{1}{2} \xi R \phi_{\xi}^{2}\right)
$$

Then, the form of the scalar field energy-momentum tensor that we find is following

$$
\begin{aligned}
T_{\mu \nu}^{(\xi)}= & \nabla_{\mu} \phi_{\xi} \nabla_{\nu} \phi_{\xi}-\frac{1}{2} g_{\mu \nu}\left(\nabla \phi_{\xi}\right)^{2}-g_{\mu \nu} V\left(\phi_{\xi}\right) \\
& +\xi\left[G_{\mu \nu} \phi_{\xi}^{2}-2 \nabla_{\mu}\left(\phi_{\xi} \nabla_{\nu} \phi_{\xi}\right)+2 g_{\mu \nu} \nabla^{\lambda}\left(\phi_{\xi} \nabla_{\lambda} \phi_{\xi}\right)\right]
\end{aligned}
$$

Note that since the above form of energy-momentum tensor has a term that depends algebraically on the Einstein tensor. By grouping all the dependence of $G_{\mu \nu}$ on the left hand side of Einstein equations we can rewrite them, alternatively, by using an effective energy-momentum tensor which is following

$$
\begin{aligned}
T_{\mu \nu}^{(\text {eff })}= & \frac{\kappa}{\kappa-\xi \phi_{\xi}^{2}}\left[\nabla_{\mu} \phi_{\xi} \nabla_{\nu} \phi_{\xi}-\frac{1}{2} g_{\mu \nu}\left(\nabla \phi_{\xi}\right)^{2}-g_{\mu \nu} V\left(\phi_{\xi}\right)\right. \\
& \left.-\xi\left[2 \nabla_{\mu}\left(\phi_{\xi} \nabla_{\nu} \phi_{\xi}\right)-2 g_{\mu \nu} \nabla^{\lambda}\left(\phi_{\xi} \nabla_{\lambda} \phi_{\xi}\right)\right]\right], \kappa=\frac{1}{8 \pi G}
\end{aligned}
$$

This is the relevant expression for the our analysis of the null energy condition.

Like earlier we arrive at the following expression for the NEC, considering an arbitrary null vector $n^{\mu}$ 


$$
\begin{aligned}
& T_{\mu \nu}^{(\mathrm{eff})}(x) n^{\mu} n^{\nu} \\
& =\frac{\kappa}{\kappa-\xi \phi_{\xi}^{2}(x)}\left[\left(n^{\mu} \nabla_{\mu} \phi_{\xi}(x)\right)^{2}-2 \xi n^{\mu} n^{\nu} \nabla_{\mu}\left(\phi_{\xi}(x) \nabla_{\nu} \phi_{\xi}(x)\right)\right] \\
& =\frac{\kappa}{\kappa-\xi \phi_{\xi}^{2}(x)}\left[\phi_{\xi}^{\prime 2}(x)-\xi\left(\phi_{\xi}^{2}(x)\right)^{\prime \prime}\right]
\end{aligned}
$$

where prime denotes $\phi_{\xi}^{\prime}=n^{\mu} \nabla_{\mu} \phi_{\xi}$.

Now note that for $\xi<0$ values, any local maximum of $\phi_{\xi}^{2}$ violate NEC, similarly for $\xi>0$, any local minimum of $\phi_{\xi}^{2}$ with $\phi_{\xi}^{2}<\frac{\kappa}{\xi}$ violates NEC and finally for $\xi>0$, any local maximum of $\phi_{\xi}^{2}$ with $\phi_{\xi}^{2}>\frac{\kappa}{\xi}$.

Average null energy condition (ANEC) which often people suggest as a way to get out of this violation also does not hold here [19].

Thus we have at least found a simple and apparently quite harmless scalar field theory can in many cases violate NEC. Violating all the pointwise energy conditions is particularly simple, and violating the averaged energy conditions, though more difficult, is still generically possible.

We now show another example of non-minimal coupling classical scalar field theory where action is following

$$
\mathcal{S}=-\int \sqrt{-g} \mathrm{~d}^{4} x \frac{1}{\Lambda^{2}} R_{\mu \nu} \nabla^{\mu} \phi \nabla^{v} \phi
$$

where $\Lambda$ is a length scale introduced in the action to make the factor $\frac{R_{\mu v}}{\Lambda^{2}}$ dimensionless quantity like metric.

We know Palatini identity [21]

$$
\delta R_{\sigma v}=\nabla_{\mu}\left(\delta \Gamma_{\nu \sigma}^{\mu}\right)-\nabla_{v}\left(\delta \Gamma_{\mu \sigma}^{\mu}\right)
$$

which we can further simplify and can write

$$
\delta R_{\mu \nu}=\frac{1}{2} g^{\alpha \beta}\left[\nabla_{\alpha} \nabla_{\nu} \delta g_{\mu \beta}+\nabla_{\alpha} \nabla_{\mu} \delta g_{\nu \beta}-\nabla_{\mu} \nabla_{\nu} \delta g_{\alpha \beta}-\nabla_{\alpha} \nabla_{\beta} \delta g_{\mu \nu}\right]
$$

which implies

$$
\begin{aligned}
- & \delta R_{\mu \nu} \nabla^{\mu} \phi \nabla^{v} \phi \\
= & \frac{1}{2}\left[2 \square \phi \nabla_{\mu} \nabla_{\nu} \phi+2 \nabla_{\rho} \phi \nabla^{\rho} \nabla_{\mu} \nabla_{\nu} \phi-g_{\mu \nu}\left((\square \phi)^{2}\right.\right. \\
+ & \left.\left.\nabla_{\rho} \nabla_{\sigma} \phi \nabla^{\rho} \nabla^{\sigma} \phi+\nabla_{\rho} \phi \nabla^{\rho} \nabla_{\sigma} \nabla^{\sigma} \phi+\nabla_{\rho} \phi \nabla_{\sigma} \nabla^{\rho} \nabla^{\sigma} \phi\right)\right] \delta g^{\mu v} \\
\Rightarrow T_{\mu \nu}= & -\frac{1}{2 \Lambda^{2}}\left[2 \square \phi \nabla_{\mu} \nabla_{\nu} \phi+2 \nabla_{\rho} \phi \nabla^{\rho} \nabla_{\mu} \nabla_{\nu} \phi-g_{\mu \nu}\left((\square \phi)^{2}\right.\right. \\
& \left.\left.+\nabla_{\rho} \nabla_{\sigma} \phi \nabla^{\rho} \nabla^{\sigma} \phi+\nabla_{\rho} \phi \nabla^{\rho} \nabla_{\sigma} \nabla^{\sigma} \phi+\nabla_{\rho} \phi \nabla_{\sigma} \nabla^{\rho} \nabla^{\sigma} \phi\right)\right] \\
& -g_{\mu \nu} R_{\alpha \beta} \nabla^{\alpha} \phi \nabla^{\beta} \phi
\end{aligned}
$$

Now we go back to our analysis. Here for any arbitrary null vector $n^{\mu}$ 


$$
\begin{aligned}
T_{\mu \nu} n^{\mu} n^{v} & =-\frac{1}{\Lambda^{2}} \square \phi n^{\mu} n^{\nu} \nabla_{\mu} \nabla_{\nu} \phi-\frac{1}{\Lambda^{2}} \nabla_{\rho} \phi n^{\mu} n^{\nu} \nabla^{\rho} \nabla_{\mu} \nabla_{\nu} \phi \\
& =-\frac{1}{\Lambda^{2}} \square \phi \nabla_{\vec{n}} \nabla_{\vec{n}} \phi-\frac{1}{\Lambda^{2}} \nabla_{\rho} \phi \nabla^{\rho} \nabla_{n} \nabla_{n} \phi \\
& =-\frac{1}{\Lambda^{2}} \nabla_{\rho}\left(\nabla^{\rho} \phi \nabla_{n} \nabla_{n} \phi\right)
\end{aligned}
$$

So, as we can see this is not positive definite quantity therefore, in this case also NEC can be violated by matter depending on the dynamics of real scalar field.

\subsection{Few Additional Comments}

- Most of the time violation of NEC is avoided because analysing the stability of the scalar, vector theories in minimally coupled field theories, people found that the condition for the absence of ghosts is the same as the requirement of satisfying NEC. Thus one argue based on minimal coupling actions that we cannot escape the presence of ghosts and instabilities in solutions where NEC is violated [22] [23] [24] [25] and therefore, can conclude that the violation is not healthy.

- But Analyzing above examples of non-minimally coupled field theories we saw that violation of NEC indeed can possible. Cosmolosist after studying inflation confirms that NEC violation can be possible [26] [27]. In [28] author gave a short review of scalar field theories with second-derivative Lagrangians, whose field equations are second order among which some of these theories admit solutions violating the Null Energy Condition and having no obvious pathologies.

- People also have studied that formation of trasversable of wormholes require such exotice matters which violate NEC [29] [30] [31] [32] [33].

\section{Violataion of NEC in Quantum Field Theory}

\subsection{Real Scalar Field Theory in Minkowski Spacetime}

We consider following action

$$
\mathcal{S}=-\int \mathrm{d}^{4} x \frac{1}{2} \eta^{\mu v} \partial_{\mu} \phi(x) \partial_{\nu} \phi(x)
$$

where $\eta_{\mu v}=\operatorname{diag}(-1,1,1,1)$ and the stress-energy tensor is given by

$$
\begin{aligned}
T_{\mu \nu}(x) & =-\frac{2}{\sqrt{-g}} \frac{\delta \mathcal{S}}{\delta g^{\mu \nu}(x)} \\
& =\partial_{\mu} \phi(x) \partial_{\nu} \phi(x)-\frac{1}{2} \eta_{\mu \nu} \partial_{\lambda} \phi(x) \partial^{\lambda} \phi(x)
\end{aligned}
$$

We will not consider the part proportional to the metric. This is simply because ultimately we will contract the expectation value of the full stress tensor twice with a null vector, thus the second term on the r.h.s. will vanish.

We can write down the scalar field operator $\phi(x)$ in terms of creation, annihilation operator in the basis of solutions of Klein Gordon equation which is 
the classical equation of motion

$$
\partial_{\lambda} \partial^{\lambda} \phi(x)=0
$$

A solution to the above equation are set of plane wave of following form

$$
f_{k}(x)=\frac{1}{\sqrt{2 \omega_{k} V}} \mathrm{e}^{i\left(\boldsymbol{k} \cdot x-\omega_{k} t\right)}, \omega_{\boldsymbol{k}}=|\boldsymbol{k}|
$$

Therefore, in terms of these solutions we can write

$$
\hat{\phi}(x)=\sum_{k}\left[\hat{a}_{k} f_{k}(x)+\hat{a}_{k}^{\dagger} f_{k}^{*}(x)\right]
$$

From the equal time commutation relations between field and its conjugate momentum operator one can find that

$$
\begin{aligned}
& {\left[\hat{a}_{\boldsymbol{k}}, \hat{a}_{\boldsymbol{l}}\right]=0=\left[\hat{a}_{\boldsymbol{k}}^{\dagger}, \hat{a}_{\boldsymbol{l}}^{\dagger}\right]} \\
& {\left[\hat{a}_{\boldsymbol{k}}^{\dagger}, \hat{a}_{l}^{\dagger}\right]=\delta^{(3)}(\boldsymbol{k}-\boldsymbol{l})}
\end{aligned}
$$

We also define the vacuum as the state annihilated by annihilation operators $\hat{a}_{\boldsymbol{k}}$ and denoted by $|0\rangle$

$$
\hat{a}_{\boldsymbol{k}}|0\rangle=0, \forall \boldsymbol{k}
$$

Higher excited states can be formed by acting creation operators $\left\{\hat{a}_{k}^{\dagger}\right\}$ on the vacuum state.

From the algebra of creation, annihilation operators one can easily find the action of creation, annihilation operators on the state belong to Fock space. For example if we have a state containing $n$ particles with momentum $\boldsymbol{k}$ denoted by $\boldsymbol{n}_{\boldsymbol{k}}$ then

$$
\hat{a}_{k}\left|n_{k}\right\rangle=\sqrt{n}\left|(n-1)_{k}\right\rangle, \hat{a}_{k}^{\dagger}\left|n_{k}\right\rangle=\sqrt{n+1}\left|(n+1)_{k}\right\rangle
$$

We will compute the stress-energy tensor operator of each component of $\hat{T}_{\mu \nu}$ separately so that the negative energy density contribution becomes manifest from the start.

$$
\begin{aligned}
\hat{T}_{t t}(x)= & \sum_{k, l} \omega_{k} \omega_{l}\left[-\hat{a}_{k} \hat{a}_{l} f_{k}(x) f_{l}(x)+\hat{a}_{k} \hat{a}_{l}^{\dagger} f_{k}(x) f_{l}^{*}(x)\right. \\
& \left.+\hat{a}_{k}^{\dagger} \hat{a}_{l} f_{k}^{*}(x) f_{l}(x)-\hat{a}_{k}^{\dagger} \hat{a}_{l}^{\dagger} f_{k}^{*}(x) f_{l}^{*}(x)\right] \\
\hat{T}_{i j}(x)= & \delta_{i j} \sum_{k, l} k^{i} l^{j}\left[-\hat{a}_{k} \hat{a}_{l} f_{k}(x) f_{l}(x)+\hat{a}_{k} \hat{a}_{l}^{\dagger} f_{k}(x) f_{l}^{*}(x)\right. \\
& \left.+\hat{a}_{k}^{\dagger} \hat{a}_{l} f_{k}^{*}(x) f_{l}(x)-\hat{a}_{k}^{\dagger} \hat{a}_{l}^{\dagger} f_{k}^{*}(x) f_{l}^{*}(x)\right]
\end{aligned}
$$

where $i, j$ are not summed over.

Now we follow normal ordering to get rid of infinity coming from the vaccum expectation value of stress-energy tensor due to zero-point energy.

$$
\begin{aligned}
: \hat{T}_{t t}(x):= & \sum_{k, l} \omega_{k} \omega_{l}\left[-\hat{a}_{k} \hat{a}_{l} f_{k}(x) f_{l}(x)+\hat{a}_{k} \hat{a}_{l}^{\dagger} f_{k}(x) f_{l}^{*}(x)\right. \\
& \left.+\hat{a}_{k}^{\dagger} \hat{a}_{l} f_{k}^{*}(x) f_{l}(x)-\hat{a}_{k}^{\dagger} \hat{a}_{l}^{\dagger} f_{k}^{*}(x) f_{l}^{*}(x)\right] \\
: \hat{T}_{i j}(x):= & \delta_{i j} \sum_{k, l} k^{i} l^{j}\left[-\hat{a}_{k} \hat{a}_{l} f_{k}(x) f_{l}(x)+\hat{a}_{l}^{\dagger} \hat{a}_{k} f_{k}(x) f_{l}^{*}(x)\right. \\
& \left.+\hat{a}_{k}^{\dagger} \hat{a}_{l} f_{k}^{*}(x) f_{l}(x)-\hat{a}_{k}^{\dagger} \hat{a}_{l}^{\dagger} f_{k}^{*}(x) f_{l}^{*}(x)\right]
\end{aligned}
$$


Note that during the calculation of stress-energy tensor operator we did not consider the term proportional to $\eta_{\mu \nu}$ which is irrelevant for our purpose.

With the normal ordered version of stress-energy tensor operators, one can easily that vacuum expectation value of these operators are zero.

Now we consider a different state which is of following form

$$
|\psi\rangle=\frac{\sqrt{3}}{2}|0\rangle+\frac{1}{2}\left|2_{k}\right\rangle
$$

and w.r.t this state expectation value of stress-energy tensor operators are following

$$
\begin{aligned}
& \left\langle\psi\left|: \hat{T}_{t t}:\right| \psi\right\rangle \\
& =\frac{\sqrt{3}}{4}\left\langle 0\left|: \hat{T}_{t t}:\right| 2_{\boldsymbol{k}}\right\rangle+\frac{\sqrt{3}}{4}\left\langle 2_{\boldsymbol{k}}\left|: \hat{T}_{t t}:\right| 0\right\rangle+\frac{1}{4}\left\langle 2_{\boldsymbol{k}}\left|: \hat{T}_{t t}:\right| 2_{\boldsymbol{k}}\right\rangle \\
& =-\frac{\sqrt{6}}{4} \omega_{\boldsymbol{k}}^{2} f_{\boldsymbol{k}}^{2}(x)-\frac{\sqrt{6}}{4} \omega_{\boldsymbol{k}}^{2} f_{\boldsymbol{k}}^{* 2}(x)+\omega_{\boldsymbol{k}}^{2} f_{\boldsymbol{k}}(x) f_{\boldsymbol{k}}^{*}(x) \\
& =\frac{\omega_{\boldsymbol{k}}}{2 V}\left[1-\frac{\sqrt{6}}{2} \cos \left(\boldsymbol{k} \cdot \boldsymbol{x}-\omega_{\boldsymbol{k}} t\right)\right] \\
& \left\langle\psi\left|: \hat{T}_{i j}:\right| \psi\right\rangle=\delta_{i j} \frac{k^{i} k^{j}}{2 \omega_{\boldsymbol{k}} V}\left[1-\frac{\sqrt{6}}{2} \cos \left(\boldsymbol{k} \cdot \boldsymbol{x}-\omega_{\boldsymbol{k}} t\right)\right] \\
& \Rightarrow\left\langle\psi\left|: \hat{T}_{i i}:\right| \psi\right\rangle=\frac{\left(k^{i}\right)^{2}}{2 \omega_{\boldsymbol{k}} V}\left[1-\frac{\sqrt{6}}{2} \cos \left(\boldsymbol{k} \cdot \boldsymbol{x}-\omega_{\boldsymbol{k}} t\right)\right]
\end{aligned}
$$

We can clearly seen that for certain combination of $\left(\boldsymbol{k} \cdot \boldsymbol{x}-\omega_{\boldsymbol{k}} t\right)$ $\left\langle\psi\left|: \hat{T}_{t t}:\right| \psi\right\rangle,\left\langle\psi\left|: \hat{T}_{i i}:\right| \psi\right\rangle$ could be negative and therefore, if we consider a null vector $\boldsymbol{n}=(1,0,0,1)$ then we can clearly see that $\left\langle\psi\left|: \hat{T}_{\mu \nu}:\right| \psi\right\rangle n^{\mu} n^{\nu}$ could be negative which clearly shows the violation of NEC.

Now at this point one may ask why not we restrict ourselves to the vacuum expectation value of stress-energy tensor operator instead of considering its expectation value w.r.t an arbitrary state. The reason is although vacuum state is stable but because of having finite non-zero temperature or any external perturbation state of any system actually becomes linear combination of vacuum and higher excited states with some suitable probability distribution. That's why one should also consider NEC w.r.t these kind of states.

\subsection{Formalism in Curved Spacetime}

For an arbitrary background geometry we can write down the action for a minimally couple real scalar field theory as follows

$$
\mathcal{S}=\int \sqrt{-g(x)} \mathrm{d}^{4} x \frac{1}{2} g^{\mu \nu}(x) \partial_{\mu} \phi(x) \partial_{\nu} \phi(x)
$$

Let us consider a complete set of mode solutions $\left\{f_{j}, f_{j}^{*}\right\}$ of the KleinGordon equation coming from the minimizing the variation of action, with $\{j\}$ being a set of discrete or continuous labels distinguishing between independent solutions. These modes are normalised with respect to following inner product 


$$
\langle f, g\rangle=\int \sqrt{-g(x)} \mathrm{d}^{3} x\left[f^{*}(t, x) \overrightarrow{\partial^{0}} g(t, x)\right]
$$

such that

$$
\begin{aligned}
& \left\langle f_{j}, f_{j^{\prime}}\right\rangle=\delta_{i j^{\prime}},\left\langle f_{j}^{*}, f_{j^{\prime}}^{*}\right\rangle=-\delta_{j j^{\prime}} \\
& \left\langle f_{j}^{*}, f_{j^{\prime}}\right\rangle=\left\langle f_{j}, f_{j^{\prime}}^{*}\right\rangle=0
\end{aligned}
$$

The above inner product is well-defined since it is time-independent which one can easily check.

And the corresponding completeness relation can be written as:

$$
\begin{aligned}
& \sum_{j}\left[f_{j}(t, \boldsymbol{x}) \partial^{0} f_{j}^{*}\left(t, \boldsymbol{x}^{\prime}\right)-f_{j}^{*}(t, \boldsymbol{x}) \partial^{0} f_{j}\left(t, \boldsymbol{x}^{\prime}\right)\right] \\
& =-\frac{i}{\sqrt{-g(x)}} \delta^{(3)}\left(\boldsymbol{x}-\boldsymbol{x}^{\prime}\right)
\end{aligned}
$$

The field operator $\hat{\phi}(x)$ can be written in terms of above basis solution as follows

$$
\hat{\phi}(x)=\sum_{j}\left[\hat{a}_{j} f_{j}(x)+\hat{a}_{j}^{\dagger} f_{j}^{*}(x)\right]
$$

where using the canonical comuutation relations one can establish that

$$
\left[\hat{a}_{j}, \hat{a}_{j^{\prime}}\right]=0=\left[\hat{a}_{j}^{\dagger}, \hat{a}_{j^{\prime}}^{\dagger}\right],\left[\hat{a}_{j}, \hat{a}_{j^{\prime}}^{\dagger}\right]=\delta_{j j^{\prime}}
$$

And vacuum state $|0\rangle$ is as usual defined by

$$
\hat{a}_{j}|0\rangle=0, \forall j
$$

Applying products of creation operators $\left\{\hat{a}_{j}^{\dagger}\right\}$ to the vacuum state creates multiparticle states, which form a basis of the Fock space:

$$
j_{1} j_{2} \cdots j_{n}=(n !)^{-\frac{3}{2}} \sum_{\sigma \in S_{n}} \prod_{i=1}^{n} \hat{a}_{j_{\sigma i}}^{\dagger}|0\rangle
$$

The normalisation factor is chosen such that the vectors obey the normalisation condition:

$$
\left\langle j_{1}^{\prime} \cdots j_{m}^{\prime} \mid j_{1} \cdots j_{n}\right\rangle=\frac{\delta_{n m}}{n !} \sum_{\sigma \in S_{n}} \prod_{i=1}^{n} \delta_{j_{i}, j_{\sigma i}^{\prime}}
$$

In terms of one-particle operators, the stress-energy tensor operator can be written as follows

$$
\begin{aligned}
& \hat{T}_{\mu \nu}= \sum_{i, j}\left[\mathcal{T}_{\mu \nu}\left(f_{i}, f_{j}\right) \hat{a}_{i} \hat{a}_{j}+\mathcal{T}_{\mu \nu}\left(f_{i}, f_{j}^{*}\right) \hat{a}_{i} \hat{a}_{j}^{\dagger}\right. \\
&\left.+\mathcal{T}_{\mu \nu}\left(f_{i}^{*}, f_{j}\right) \hat{a}_{i}^{\dagger} \hat{a}_{j}+\mathcal{T}_{\mu \nu}\left(f_{i}^{*}, f_{j}^{*}\right) \hat{a}_{i}^{\dagger} \hat{a}_{j}^{\dagger}\right] \\
&: \hat{T}_{\mu \nu}:= \sum_{i, j}\left[\mathcal{T}_{\mu \nu}\left(f_{i}, f_{j}\right) \hat{a}_{i} \hat{a}_{j}+\mathcal{T}_{\mu \nu}\left(f_{i}, f_{j}^{*}\right) \hat{a}_{j}^{?} \hat{a}_{i}\right. \\
&\left.+\mathcal{T}_{\mu \nu}\left(f_{i}^{*}, f_{j}\right) \hat{a}_{i}^{\dagger} \hat{a}_{j}+\mathcal{T}_{\mu \nu}\left(f_{i}^{*}, f_{j}^{*}\right) \hat{a}_{i}^{\dagger} \hat{a}_{j}^{\dagger}\right] \\
& \mathcal{T}_{\mu \nu}\left(f_{i}, f_{j}\right)=\partial_{\mu} f_{i} \partial_{v} f_{j}
\end{aligned}
$$




\subsection{Rigidly Rotating Minkowski Spacetime}

Metric of rigidly rotating Minkowski spacetime is following

$$
\mathrm{d} s^{2}=-\left(1-\rho^{2} \Omega^{2}\right) \mathrm{d} t^{2}+2 \rho^{2} \Omega \mathrm{d} t \mathrm{~d} \varphi+\mathrm{d} \rho^{2}+\rho^{2} \mathrm{~d} \varphi^{2}+\mathrm{d} z^{2}
$$

Mode solutions of Klein-Gordon equation in this background spacetime derived in [34] [35] are following

$$
f_{\omega k m}(x)=\frac{1}{\sqrt{8 \pi^{2}|\omega|}} \mathrm{e}^{-i \tilde{\omega}_{m} t+i k z+i m \varphi} J_{m}(q \rho), \tilde{\omega}_{m}=\omega-m \Omega
$$

where $q=\sqrt{\omega^{2}-k^{2}}$ and $\left\{J_{m}(q \rho)\right\}$ s are the bessel functions with argument $q \rho$.

The inner-product is

$$
\langle f, g\rangle=\int_{-\infty}^{\infty} \mathrm{d} z \int_{0}^{\infty} \rho \mathrm{d} \rho \int_{0}^{2 \pi} \mathrm{d} \varphi f\left(i \overleftrightarrow{\partial_{t}}+i \Omega \overleftrightarrow{\partial_{\varphi}}\right) g
$$

And the field operator is given by

$$
\hat{\phi}(x)=\sum_{m=-\infty}^{\infty} \int_{0}^{\infty} \omega \mathrm{d} \omega \int_{-\omega}^{\omega} \mathrm{d} k\left[\hat{a}_{k \omega m} f_{k \omega m}(x)+\hat{a}_{k \omega m}^{\dagger} f_{k \omega m}^{*}(x)\right]
$$

and the vacuum state $|0\rangle$ is defined by

$$
\hat{a}_{k \omega m}|0\rangle=0
$$

Here also we will find that $\left\langle 0\left|: \hat{T}_{\mu \nu}:\right| \psi\right\rangle=0$ and if we take as earlier a state $|\psi\rangle=\frac{\sqrt{3}}{2}|0\rangle+\frac{1}{2}\left|2_{\omega k m}\right\rangle$, we will find that

$$
\begin{aligned}
&\left\langle\psi\left|: \hat{T}_{t t}(x):\right| \psi\right\rangle \frac{\sqrt{3}}{4}\left\langle 0\left|: \hat{T}_{t t}(x):\right| 2_{\omega k m}\right\rangle+\frac{\sqrt{3}}{4}\left\langle 2_{\omega k m}\left|: \hat{T}_{t t}(x):\right| 0\right\rangle \\
&+\frac{1}{4}\left\langle 2_{\omega k m}\left|: \hat{T}_{t t}(x):\right| 2_{\omega k m}\right\rangle \\
&=-\frac{\sqrt{6}}{4} \tilde{\omega}_{m}^{2} f_{\omega k m}^{2}(x)-\frac{\sqrt{6}}{4} \tilde{\omega}_{m}^{2} f_{\omega k m}^{* 2}(x)+\tilde{\omega}_{m}^{2}\left|f_{\omega k m}(x)\right|^{2} \\
&= \frac{\tilde{\omega}_{m}^{2}}{8 \pi^{2}|\omega|} J_{m}^{2}(q \rho)\left[1-\frac{\sqrt{6}}{2} \cos \left(k z+m \varphi-\tilde{\omega}_{m} t\right)\right] \\
&\left\langle\psi\left|: \hat{T}_{z z}(x):\right| \psi\right\rangle=\frac{k^{2}}{8 \pi^{2}|\omega|} J_{m}^{2}(q \rho) \times\left[1-\frac{\sqrt{6}}{2} \cos \left(k z+m \varphi-\tilde{\omega}_{m} t\right)\right]
\end{aligned}
$$

Note that both $\left\langle\psi\left|: \hat{T}_{t t}(x):\right| \psi\right\rangle, \psi: \hat{T}_{z z}(x): \psi$ can be negative for a certain combinations of $k z+m \varphi-\tilde{\omega}_{m} t$.

Now we choose a null vector $\boldsymbol{n}=\left(1,0,0, \sqrt{1-\rho^{2} \Omega^{2}}\right)$ then

$$
\begin{aligned}
\left\langle\psi\left|: \hat{T}_{\mu \nu}(x):\right| \psi\right\rangle n^{\mu} n^{v} \\
=\left\langle\psi\left|: \hat{T}_{t t}(x):\right| \psi\right\rangle+\left\langle\psi\left|: \hat{T}_{z z}(x):\right| \psi\right\rangle\left(1-\rho^{2} \Omega^{2}\right) \\
=\frac{k^{2}\left(1-\rho^{2} \Omega^{2}\right)+\tilde{\omega}_{m}^{2}}{8 \pi^{2}|\omega|} J_{m}^{2}(q \rho)\left[1-\frac{\sqrt{6}}{2} \cos \left(k z+m \varphi-\tilde{\omega}_{m} t\right)\right]
\end{aligned}
$$


Note that the prefactor solely depends on the coordinate $\rho$ and if we choose $\rho<\frac{1}{\Omega}$, then we can see that depending on the value of $k z+m \varphi-\tilde{\omega}_{m} t$ we will get wither positive or negative answer which shows that for certain spacetime points NEC condition does not hold.

Note that the state $|\psi\rangle$ that we have chosen to show that in Quantum field theory NEC is indeed violated, is nothing special. One can choose a certain class of state which are linear combinations of vacuum and excited states w.r.t which one can show that NEC is indeed violated and these states can be thought of quantum state of the system which can be thought as perturbation around vacuum because of some external perturbations like temperature and other sources.

\section{Conclusions}

Quantum version of NEC is violated in QFT. In many cases, the NEC violating states are superpositions, whose interference takes the form of an oscillatory term responsible for the violation. However, not all representatives of these classes violate NEC. Whether the oscillatory term leads to violations of NEC or not, depends on the normalization of the state; an example where this becomes apparent is the vacuum +2 particles states, for which exactly half of the phase space covered by these states gives rise to NEC violations and the other half does not [36].

Note that violation of NEC in QFT certainly shows that laws of black hole mechanics are not certainly valid in Quantum domain. Often people compare black hole with a thermodyncamical macroscopic system with first law of black hole mechanics to be identified with first law of thermodynamics and second law of black hole mechanics is identified with second law of thermodynamics. This can't be the case if the microscopic states of the matter which form the geometry itself violate the NEC. Even we have found there are certain class of classical matter which can also violate NEC therefore, in presence of such matter in classical background also enforce that we can't apply laws of black-hole mechanics.

Apart from violation of NEC, we found that there must be an inequality in QFT in curved spacetime for different classical backgrounds which put restriction on the state of matter based on which we can make certain comments on different physical processes. So far, there exists no bound in QFT that allows us to generalize all the theorems in General Relativity and exclude Wormholes and other exotic spacetimes. Quantum NEC is a local QFT bound that does not restrict the amount by which NEC is violated enough to do the above.

\section{Acknowledgements}

Author wants to thank Gopal Sardar for helpful discussion regarding the subject matter and his comments on the idea of this paper. Author would also like to thank CSIR to support this work through JRF fellowship. 


\section{Conflicts of Interest}

The author declares no conflicts of interest regarding the publication of this paper.

\section{References}

[1] Visser, M. (1995) Lorentzian Wormholes: From Einstein to Hawking.

[2] Flanagan, E.E and Wald, R.M. (1996) Does Back Reaction Enforce the Averaged Null Energy Condition in Semiclassical Gravity? Physical Review D, 54, 6233. https://doi.org/10.1103/PhysRevD.54.6233

[3] Barcelo, C. and Visser, M. (2000) Scalar Fields, Energy Conditions and Traversable Wormholes. Classical and Quantum Gravity, 17, 3843. https://doi.org/10.1088/0264-9381/17/18/318

[4] Hawking, S.W. and Ellis, G.F.R. (1973) The Large Scale Structure of Space-Time. Vol. 1, Cambridge University Press.

[5] Lock, M.P.E. and Fuentes, I. (2017) Dynamical Casimir Effect in Curved Spacetime. New Journal of Physics, 19, 073005. https://doi.org/10.1088/1367-2630/aa7651

[6] Saharian, A.A. (2001) Scalar Casimir Effect for D-Dimensional Spherically Symmetric Robin Boundaries. Physical Review D, 63, 125007. https://doi.org/10.1103/PhysRevD.63.125007

[7] Kandrup, H.E. (1992) Violations of the Strong Energy Condition for Interacting Systems of Particles. Physical Review D, 46, 5360. https://doi.org/10.1103/PhysRevD.46.5360

[8] Xiong, H.H. and Zhu, J.Y. (2007) Violation of Strong Energy Condition in Effective Loop Quantum Cosmology. International Journal of Modern Physics A, 22, 3137 3146. https://doi.org/10.1142/S0217751X07036658

[9] Dowker, F. (2013) Black Holes. Imperial College London, MSc Quantum Fields and Fundamental Forces, Lecture Notes.

[10] Gourgoulhon, É. (2018) Geometry and Physics of Black Holes Lecture Notes. Les Houches.

[11] Reall, H. (2014) Part 3 Black Holes. Lecture Notes Given as Part of the Cambridge University Mathematical Tripos.

[12] Adamson, R. Black Holes and Thermodynamics.

[13] Wald, R.M. (1984) General Relativity. Chicago University Press, Chicago. https://doi.org/10.7208/chicago/9780226870373.001.0001

[14] Kar, S. and Sengupta, S. (2007) The Raychaudhuri Equations: A Brief Review. Pramana, 69, 49-76. https://doi.org/10.1007/s12043-007-0110-9

[15] Sjøstrøm, Dag-Morten. Bosons and Fermions in Curved Spacetime. Master's thesis, Institutt for fysikk, 2013.

[16] Myrzakulov, R., Sebastiani, L. and Zerbini, S. (2013) Inhomogeneous Viscous Fluids in a Friedmann-Robertson-Walker (FRW) Universe. Galaxies, 1, 83-95. https://doi.org/10.3390/galaxies1020083

[17] Sebastiani, L. (2010) Dark Viscous Fluid Coupled with Dark Matter and Future Singularity. The European Physical Journal C, 69, 547-553. https://doi.org/10.1140/epjc/s10052-010-1398-z

[18] Nojiri, S. and Odintsov, S.D. (2005) Inhomogeneous Equation of State of the Universe: Phantom Era, Future Singularity, and Crossing the Phantom Barrier. Physi- 
cal Review D: Particles and Fields, 72, Article ID: 023003.

[19] Barceló, C. and Visser, M. (2000) Scalar Fields, Energy Conditions and Traversable Wormholes. Classical and Quantum Gravity, 17, 3843-3864. https://doi.org/10.1088/0264-9381/17/18/318

[20] Ford, L.H. and Roman, T.A. (2001) Classical Scalar Fields and the Generalized Second Law. Physical Review D, 64, Article ID: 024023. https://doi.org/10.1103/PhysRevD.64.024023

[21] Weinberg, S. (1972) Gravitation and Cosmology: Principles and Applications of the General Theory of Relativity. Wiley, New York.

[22] Dubovsky, S., Grégoire, T., Nicolis, A. and Rattazzi, R. (2006) Null Energy Condition and Superluminal Propagation. Journal of High Energy Physics, No. 3, 025.

[23] Buniy, R.V., Hsu, S.D.H. and Murray, B.M. (2006) The Null Energy Condition and Instability. Physical Review D, 74, Article ID: 063518.

[24] Buniy, R.V. and Hsu, S.D.H. (2006) Instabilities and the Null Energy Condition. Physics Letters B, 632, 543-546. https://doi.org/10.1016/j.physletb.2005.10.075

[25] Elder, B., Joyce, A. and Khoury, J. (2014) From Satisfying to Violating the Null Energy Condition. Physical Review D, 89, Article ID: 044027. https://doi.org/10.1103/PhysRevD.89.044027

[26] Winitzki, S. (2001) Null Energy Condition Violations in Eternal Inflation. arXiv preprint gr-qc/0111109

[27] Baldi, M., Finelli, F. and Matarrese, S. (2005) Inflation with Violation of the Null Energy Condition. Physical Review D, 72, Article ID: 083504.

https://doi.org/10.1103/PhysRevD.72.083504

[28] Rubakov, V.A. (2014) The Null Energy Condition and Its Violation. Physics-Uspekhi, 57, 128. https://doi.org/10.3367/UFNe.0184.201402b.0137

[29] Visser, M. (2008) Traversable Wormholes from Surgically Modified Schwarzschild Spacetimes. arXiv preprint arXiv:0809.0927

[30] Visser, M., Kar, S. and Dadhich, N. (2003) Traversable Wormholes with Arbitrarily Small Energy Condition Violations. Physical Review Letters, 90, Article ID: 201102. https://doi.org/10.1103/PhysRevLett.90.201102

[31] Barcelo, C. and Visser, M. (1999) Traversable Wormholes from Massless Conformally Coupled Scalar Fields. Physics Letters B, 466, 127-134. https://doi.org/10.1016/S0370-2693(99)01117-X

[32] Kar, S., Dadhich, N. and Visser, M. (2004) Quantifying Energy Condition Violations in Traversable Wormholes. Pramana, 63, 859-864. https://doi.org/10.1007/BF02705207

[33] Barceló, C. and Visser, M. (2000) Brane Surgery: Energy Conditions, Traversable Wormholes, and Voids. arXiv preprint hep-th/0004022

[34] Ambrus, V.E. (2014) Dirac Fermions on Rotating Space-Times. PhD Thesis, University of Sheffield, Sheffield.

[35] Ambrus, V.E. and Winstanley, E. (2014) Rotating Quantum States. Physics Letters $B$, 734, 296-301. https://doi.org/10.1016/j.physletb.2014.05.031

[36] Pfenning, M.J. (1998) Quantum Inequality Restrictions on Negative Energy Densities in Curved Spacetimes. PhD Thesis, Tufts University, Medford. 